\title{
Single-top production and rare top interactions
}

\author{
Pratishruti Saha, ${ }^{1, *}$ Ken Kiers, ${ }^{2, \dagger}$ and Alejandro Szynkman ${ }^{3, \$}$ \\ ${ }^{1}$ Harish-Chandra Research Institute, Chhatnag Road, Jhunsi, Allahabad-211019, India \\ ${ }^{2}$ Physics and Engineering Department, Taylor University, \\ 236 West Reade Avenue, Upland, Indiana 46989, USA \\ ${ }^{3}$ IFLP, CONICET-Dpto. de Física, Universidad Nacional de La Plata, C.C. 67, 1900 La Plata, Argentina
}

(Received 10 January 2018; published 1 August 2018)

\begin{abstract}
The study of the top quark's properties is an important part of the LHC program. In earlier work, we have studied the rare decay $t \rightarrow b \bar{b} c$, using effective operators to capture the effects of physics beyond the Standard Model. However top decay is primarily sensitive to new physics in the sub-TeV energy regime. If this new physics resides at a higher energy scale, then one needs to turn to single-top production. In this paper, we use the $s$-channel and $t$-channel single-top production measurements to constrain the new physics parameter space associated with such contact interactions. We also study the net top polarization as a means to distinguish between contributions from operators involving different fermion chiralities and Lorentz structures.
\end{abstract}

DOI: 10.1103/PhysRevD.98.035003

\section{INTRODUCTION}

The top quark has long been believed to be colluding with new physics (NP). However, intense scrutiny of the top quark's properties at the Tevatron and at the LHC has so far not revealed any conclusive departures from the Standard Model (SM). For a few years, the Tevatron experiments reported a large forward-backward asymmetry in top pair production. With the accumulation of more statistics and improved calculation of the SM predictions, however, this anomaly disappeared [1]. Nevertheless, the top quark remains a likely suspect: its mass differs from those of other SM fermions by orders of magnitude, so much so that it threatens to push the electroweak vacuum beyond the edge of stability. Moreover, several anomalies in the $B$ sector [2-5] continue to fuel speculations that third-generation quarks may be the much-sought window to physics beyond the SM.

In earlier work [6], we proposed a study of rare decay modes of the top quark. Since all the top quark measurements to date have been made in channels involving the dominant decay modes of the top, they would, naturally, have missed signs of new physics that only manifest in the rare decay modes. We examined the sensitivity of the LHC

\footnotetext{
*pratishrutisaha@hri.res.in

"knkiers@taylor.edu

*szynkman@ fisica.unlp.edu.ar
}

Published by the American Physical Society under the terms of the Creative Commons Attribution 4.0 International license. Further distribution of this work must maintain attribution to the author(s) and the published article's title, journal citation, and DOI. Funded by SCOAP . to the rare decay $t \rightarrow b \bar{b} c$ and found that with $3000 \mathrm{fb}^{-1}$ of data, the LHC would be able to set statistically significant limits on such decays. However, it is evident that top decay would be most sensitive to new physics effects arising at the energy scale of a few hundred $\mathrm{GeV}$ at most. If the new physics contributions originate at higher energy scales, the impact on top decay would be too small to be discernible. In order to probe such interactions further, one must turn to single-top production.

In Ref. [6], NP contributions to the rare decay $t \rightarrow b \bar{b} c$ were parametrized in terms of various four-Fermi operators. In this paper, we examine the impact of that same set of operators on single-top production. While the top quark decay in the mode $t \rightarrow b \bar{b} c$ has not been observed, singletop production has been measured and these measurements can, in principle, be used to constrain the strength of these interactions. A priori, it may seem that the contribution of such operators to single-top production would be diminished by the parton densities of the heavy quarks in the initial state. While this is true in general, the situation is salvaged somewhat by the fact that the competing SM mode is driven by electroweak interactions and not by strong interactions. A detailed numerical study shows that it is possible to set meaningful limits on the parameters of the interactions using existing LHC data. We also present a futuristic scenario in which very stringent limits may be obtained. This possibility, however, is contingent upon the development of reliable techniques to determine the charge of an outgoing $b$ quark on an event-by-event basis. We further examine the possibility of distinguishing between the contributions of new physics operators with different Lorentz structures and fermion chiralities using the polarization of the top quark. 
Single top production has received significant attention as a direct probe of physics beyond the SM [7,8]. Since the present analysis focuses on the effects of four-quark operators, it is worth mentioning that such operators have been studied quite extensively, particularly in the context of flavor-changing neutral currents involving the top quark [9].

The remainder of this paper is organized as follows. In Sec. II, we discuss the theoretical generalities related to single-top production at the LHC and introduce the effective operators that we use to parametrize new physics contributions, though the detailed analytic expressions are consigned to the Appendix. In Sec. III, we discuss our numerical analyses and results for $s$-channel as well as $t$-channel single-top production at the LHC at centerof-mass energies of 8 and $13 \mathrm{TeV}$. We conclude in Sec. IV.

\section{SINGLE TOP PRODUCTION}

At a hadron collider, the dominant production mode for top quarks is $p p(p \bar{p}) \rightarrow t \bar{t}$. Single top production is subdominant. Nonetheless, it is important as it provides a cleaner way of measuring the electroweak couplings of the top quark. Within the framework of the SM, single-top production at hadron colliders is classified into three production channels as shown in Fig. 1, namely, $s$ channel, $t$ channel and $W t$ channel. At the LHC, $t$-channel production is the dominant mode, followed by $W t$-associated production. Cross sections for all three channels have been measured at the LHC during the 7 and $8 \mathrm{TeV}$ runs [10-16]. The $13 \mathrm{TeV}$ run is ongoing and results are already available in some channels [17,18]. These are summarized in Fig. 2. For $t$-channel single-top production some kinematic

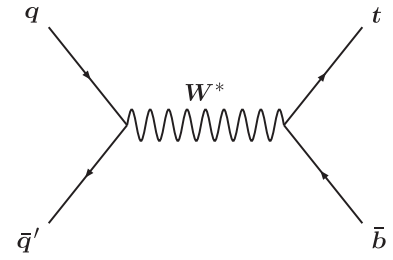

(a)

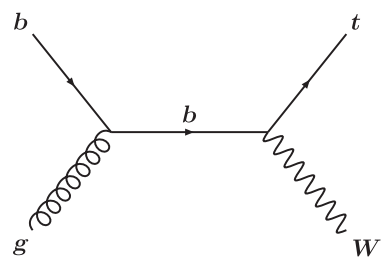

(c)

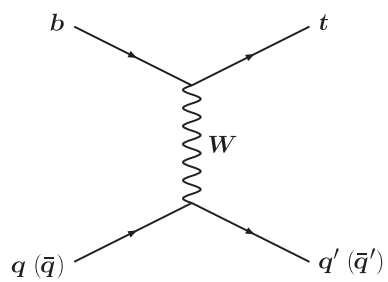

(b)

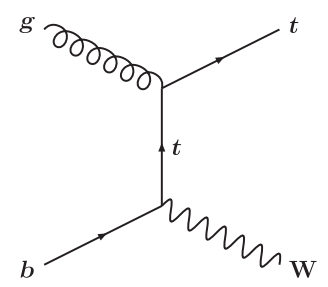

(d)
FIG. 1. (a) $s$-channel, (b) $t$-channel, and (c)-(d) $W t$-channel single-top production modes. distributions have also been measured [11,19,20]. All the measurements are, by and large, in agreement with the SM predictions, even though certain channels are plagued by large experimental uncertainties.

\section{A. Effective Lagrangian}

In Ref. [6], the contributions from physics beyond the SM to the rare decay $t \rightarrow b \bar{b} c$ were parametrized in terms of the six-dimensional operators given by $\mathcal{L}_{\text {eff }}$,

$$
\mathcal{L}_{\text {eff }}=\mathcal{L}_{\text {eff }}^{V}+\mathcal{L}_{\text {eff }}^{S}+\mathcal{L}_{\text {eff }}^{T},
$$

where

$$
\begin{aligned}
\mathcal{L}_{\text {eff }}^{V}= & 4 \sqrt{2} G_{F} V_{c b} V_{t b}\left\{X_{L L}^{V} \bar{b} \gamma_{\mu} P_{L} t \bar{c} \gamma^{\mu} P_{L} b\right. \\
& +X_{L R}^{V} \bar{b} \gamma_{\mu} P_{L} t \bar{c} \gamma^{\mu} P_{R} b+X_{R L}^{V} \bar{b} \gamma_{\mu} P_{R} t \bar{c} \gamma^{\mu} P_{L} b \\
& \left.+X_{R R}^{V} \bar{b} \gamma_{\mu} P_{R} t \bar{c} \gamma^{\mu} P_{R} b\right\}+ \text { H.c., } \\
\mathcal{L}_{\text {eff }}^{S}= & 4 \sqrt{2} G_{F} V_{c b} V_{t b}\left\{X_{L L}^{S} \bar{b} P_{L} t \bar{c} P_{L} b+X_{L R}^{S} \bar{b} P_{L} t \bar{c} P_{R} b\right. \\
& \left.+X_{R L}^{S} \bar{b} P_{R} t \bar{c} P_{L} b+X_{R R}^{S} \bar{b} P_{R} t \bar{c} P_{R} b\right\}+ \text { H.c., } \\
\mathcal{L}_{\text {eff }}^{T}= & 4 \sqrt{2} G_{F} V_{c b} V_{t b}\left\{X_{L L}^{T} \bar{b} \sigma^{\mu \nu} P_{L} t \bar{c} \sigma_{\mu \nu} P_{L} b\right. \\
& \left.+X_{R R}^{T} \bar{b} \sigma^{\mu \nu} P_{R} t \bar{c} \sigma_{\mu \nu} P_{R} b\right\}+ \text { H.c. }
\end{aligned}
$$

Clearly, these operators can also contribute to singletop production. The $W t$ channel would remain unaffected by these NP contributions. However, final states identical to those produced in $s$-channel and $t$-channel processes can occur via the contact interactions listed above.

\section{B. Contribution to single-top production from $\mathcal{L}_{\text {eff }}$}

The operators listed in Eqs. (1)-(3) can give rise to three possible amplitudes for single top production: $\bar{b} c \rightarrow t \bar{b}, b c \rightarrow t b$, and $b \bar{b} \rightarrow t \bar{c}$. In the SM, the first one is an $s$-channel process, whereas the second and third are $t$-channel processes. In addition, the three final states get contributions from light-quark initial states in the SM. Some of the key features are as follows:

(i) For single-top production due to such operators, the initial states would necessarily consist of bottom and charm quarks. The low densities of these inside the proton tend to suppress the cross section as compared to the SM production rates. This effect is more pronounced in the $s$ channel than in the $t$ channel as the SM rate for the latter is CabibboKobayashi-Maskawa (CKM) suppressed unless there is a $b$ quark in the initial state (see Fig. 1).

(ii) The suppression caused by the low initial-state parton densities is compensated to some extent by the growth in the cross section with increase in the 


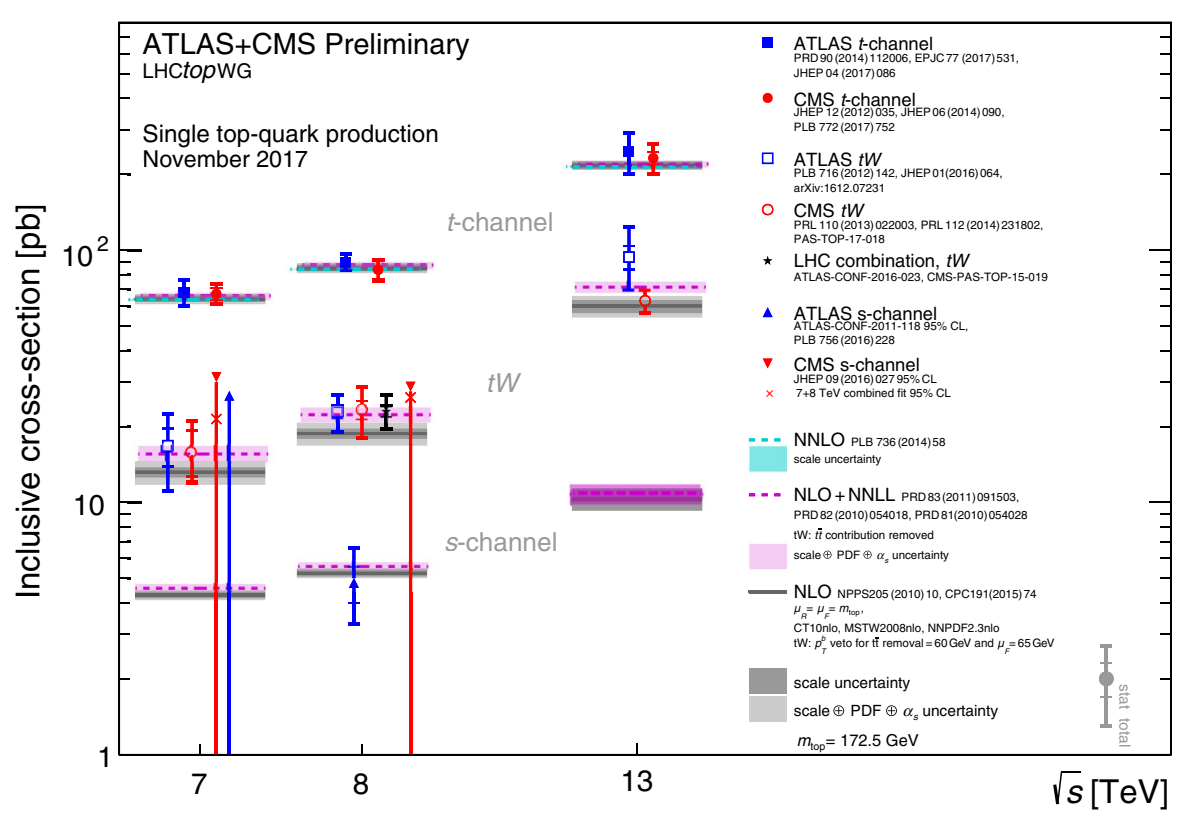

FIG. 2. Summary of single-top production rates measured at the LHC [21].

parton c.m. energy $(\sqrt{\hat{s}})$. This, of course, is a generic feature of contact interactions.

(iii) When new physics is parametrized in terms of effective operators, the implicit assumption is that the operators arise due to physics at a very high energy scale $\left(\Lambda_{\text {new }}\right)$ that is beyond the direct reach of current experiments. When the operators listed in Eqs. (1)-(3) are applied to top decay, $\Lambda_{\text {new }} \sim$ $\mathcal{O}(\mathrm{TeV})$ is permissible, given that the energy scale of the interaction is $m_{t} \sim 173 \mathrm{GeV}$. However, in the context of single-top production in $p p$ collisions at LHC energies, one must necessarily consider significantly larger values of $\Lambda_{\text {new }} \sim \mathcal{O}(10 \mathrm{TeV})$ or higher. In the parametrization used in this work and in our previous work on this topic [6], $\Lambda_{\text {new }} \sim\left(G_{F} X_{A B}^{I}\right)^{-1 / 2}$. Consequently, $X_{A B}^{I} \sim \mathcal{O}(1)$ correspond to $\Lambda_{\text {new }}$ in the sub-TeV regime. In principle, it should be possible to rule out such new physics scenarios as they could lead to resonances in the $s$-channel production mode, causing a spike in the cross section. However, owing to the relatively large uncertainty in the measured $s$-channel cross section, no robust conclusions can be drawn at this stage. In the $t$ channel, such contributions would manifest, for example, in the form of a harder transverse momentum distribution. However, no such deviations have been found up to $p_{T} \sim 300 \mathrm{GeV}[11,19,20]$.

In our previous work related to $t \rightarrow b \bar{b} c$, the ten couplings $X_{A B}^{I}$ from the NP effective Lagrangian $\mathcal{L}_{\text {eff }}$ were found to appear together in six characteristic combinations. We denoted these six combinations by $\hat{A}_{i}^{\sigma}$, defining

$$
\begin{aligned}
& \hat{A}_{\bar{b}}^{+}=4\left|X_{L L}^{V}\right|^{2}-8 \operatorname{Re}\left(X_{L L}^{T} X_{L L}^{S *}\right)+32\left|X_{L L}^{T}\right|^{2}, \\
& \hat{A}_{\bar{b}}^{-}=4\left|X_{R R}^{V}\right|^{2}-8 \operatorname{Re}\left(X_{R R}^{T} X_{R R}^{S *}\right)+32\left|X_{R R}^{T}\right|^{2}, \\
& \hat{A}_{b}^{+}=\left|X_{L L}^{S}\right|^{2}+\left|X_{L R}^{S}\right|^{2}-16\left|X_{L L}^{T}\right|^{2}, \\
& \hat{A}_{b}^{-}=\left|X_{R R}^{S}\right|^{2}+\left|X_{R L}^{S}\right|^{2}-16\left|X_{R R}^{T}\right|^{2}, \\
& \hat{A}_{c}^{+}=4\left|X_{L R}^{V}\right|^{2}+8 \operatorname{Re}\left(X_{L L}^{T} X_{L L}^{S *}\right)+32\left|X_{L L}^{T}\right|^{2}, \\
& \hat{A}_{c}^{-}=4\left|X_{R L}^{V}\right|^{2}+8 \operatorname{Re}\left(X_{R R}^{T} X_{R R}^{S *}\right)+32\left|X_{R R}^{T}\right|^{2} .
\end{aligned}
$$

In addition to depending on the above six quantities, various observables were also found to depend on the real and imaginary parts of $X_{L L}^{V}$ and on the combination $\operatorname{Im}\left(X_{L L}^{T} X_{L L}^{S *}+X_{R R}^{T} X_{R R}^{S *}\right){ }^{1}$

In the case of single-top production, once again, NP contributions to the various cross sections of interest can be expressed in terms of these same combinations of NP parameters. Explicit expressions for the matrix elements squared in the different cases may be found in the Appendix. If we restrict our attention to the case in which we sum over the top quark's spin, we find that the 20-dimensional parameter space spanned by the ten complex-valued parameters $X_{A B}^{I}$ is reduced to a fivedimensional parameter space composed of $\left(\hat{A}_{\bar{b}}^{+}+\hat{A}_{\bar{b}}^{-}\right)$, $\left(\hat{A}_{b}^{+}+\hat{A}_{b}^{-}\right),\left(\hat{A}_{c}^{+}+\hat{A}_{c}^{-}\right), \operatorname{Re}\left(X_{L L}^{V}\right)$ and $\operatorname{Im}\left(X_{L L}^{V}\right){ }^{2}$ If one wishes to consider top quark polarization effects, there

\footnotetext{
${ }^{1}$ The dependence on $X_{L L}^{V}$ comes from SM-NP interference terms. Terms proportional to $\operatorname{Im}\left(X_{L L}^{T} X_{L L}^{S *}+X_{R R}^{T} X_{R R}^{S *}\right)$ are associated with triple-product correlations [6].

${ }^{2}$ As we have noted in past work, the situation is somewhat complicated by the fact that the real and imaginary parts of $X_{L L}^{V}$ also appear in the parameter $\hat{A}_{\bar{b}}^{+}$.
} 
TABLE I. $\quad K$ factors for $s$ - and $t$-channel single-top production cross sections at 8 and $13 \mathrm{TeV}$.

\begin{tabular}{llll}
\hline \hline & \multicolumn{1}{c}{$\sigma_{\text {NNLO }}$} & $\sigma_{\text {LO }}$ & $K$ \\
\hline$s$ channel; 8 TeV & From Ref. [26] & From MADGRAPH5 using MSTw2008LO pdfs & 1.74 \\
$s$ channel; 13 TeV & From Ref. [26] & From MADGRAPH5 using MSTW2008LO pdfs & 1.73 \\
$t$ channel; 8 TeV & From Ref. [27] & From MADGRAPH5 using MSTW2008LO pdfs & 1.06 \\
$t$ channel; 13 TeV & From Ref. [18] & From MADGRAPH5 using MSTw2008LO pdfs & 1.02 \\
\hline \hline
\end{tabular}

is an additional dependence on $\left(\hat{A}_{\bar{b}}^{+}-\hat{A}_{\bar{b}}^{-}\right),\left(\hat{A}_{b}^{+}-\hat{A}_{b}^{-}\right)$, $\left(\hat{A}_{c}^{+}-\hat{A}_{c}^{-}\right.$), and $\operatorname{Im}\left(X_{L L}^{T} X_{L L}^{S *}+X_{R R}^{T} X_{R R}^{S *}\right)$ (please refer to the Appendix for details). In the present work we shall assume that $X_{L L}^{V}$ and $X_{L L}^{T} X_{L L}^{S *}+X_{R R}^{T} X_{R R}^{S *}$ are both real, thereby reducing the size of the parameter space somewhat. ${ }^{3}$ In our numerical work below, we will mostly consider the case in which the polarization of the top quark is ignored (Secs. III A, III B, III C and III E). A polarization-dependent asymmetry is considered in Sec. IIID. Furthermore, throughout the remainder of this paper, we will consider operators with a single Lorentz structure at a time. We will also make certain assumptions about the relative magnitudes of the couplings associated with operators having the same Lorentz structure but different chirality structure.

We close this section with the following comment. The careful reader might have noticed that the effective operators listed in Eqs. (1)-(3) are not SM gauge invariant. Within a gauge-invariant framework, the presence of new operators, potentially constrained by other observables, could limit the size of the Wilson coefficients corresponding to the operators in our basis. We have examined the gauge-invariant operators involving the second and third quark generations and have found that the observables that they may directly affect are bottom and top pair production. As is the case for single-top production within our framework, the new operators lead to effects suppressed by the parton distribution functions (PDFs) of the initial heavy quarks. However, for bottom and top pair production the contributions from the new operators must also compete with the dominant QCD contributions. Therefore we expect these processes to be far less constraining than those considered in the present work.

\section{NUMERICAL STUDY}

In order to extract limits on the $\hat{A}_{i}^{\sigma}$ 's (and consequently on $X_{A B}^{I}$ ), we start by implementing $\mathcal{L}_{\text {eff }}$ alongside the Standard Model in MadGraph5 [22] using FeynRules [23]. This puts us in a position to calculate the tree-level cross section for single-top production in $p p$ collisions, for which we use CTEQ6L PDFs [24], setting both the renormalization and factorization scales to be $m_{t}=$ $173 \mathrm{GeV}$. In order to approximate higher-order QCD

\footnotetext{
${ }^{3}$ Note that $\operatorname{Im}\left(X_{L L}^{V}\right)$ plays an important role in partial rate asymmetries [6]; we neglect such effects here.
}

corrections, we estimate $K$ factors as $\sigma_{\mathrm{NNLO}}^{\mathrm{SM}}($ approx $) /$ $\sigma_{\mathrm{LO}}^{\mathrm{SM}}$, where $\sigma_{\mathrm{NNLO}}^{\mathrm{SM}}$ (approx) is obtained from the references listed in Table I and $\sigma_{\mathrm{LO}}^{\mathrm{SM}}$ is calculated using MADGRAPH5 in conjunction with MSTW2008LO PDFs [25]. ${ }^{4}$ We then compute the tree-level cross sections, including both SM and NP effects, and multiply the results by the corresponding $K$ factors to obtain estimates of the QCD-corrected values. In the following, " $\sigma_{\mathrm{SM}}$ " denotes the SM cross sections obtained in this manner.

\section{A. $t$-channel single-top production}

At the LHC, $t$-channel processes yield the dominant contribution to single-top production. These processes consist of $d_{i} u_{j} \rightarrow t d_{k}$ and $d_{i} \bar{d}_{j} \rightarrow t \bar{u}_{k}$ where $d_{i, j, k} \in$ $\{d, s, b\}$ and $u_{j, k} \in\{u, c\}$. Within the SM, the relative magnitudes of the contributions from the different initial states are governed by the densities of the respective partons inside the proton and the CKM factors appearing in the amplitude. Once $\mathcal{L}_{\text {eff }}$ is introduced, there are additional contributions to $b c \rightarrow t b$ and $b \bar{b} \rightarrow t \bar{c}$. Figure 3 shows the cross section. As noted above, the operators in $\mathcal{L}_{V}, \mathcal{L}_{S}$ and $\mathcal{L}_{T}$ are considered separately in our analysis. It can be seen that the tensor operators are constrained most tightly, followed by vector and scalar operators. This is expected, given the structure of the $\hat{A}_{i}^{\sigma}$ 's and the fact that large numerical factors accompany $X_{A B}^{T}$ wherever they appear. It is intriguing to note that, except in the case of $X_{A B}^{T}$, couplings of $\mathcal{O}(1)$ are not excluded by the experimental data at $8 \mathrm{TeV}$. How do we reconcile this with a) our earlier statement that $X_{A B}^{I} \sim \mathcal{O}(1)$ correspond to $\Lambda_{\text {new }}$ in the sub-TeV range, and $b$ ) the fact that no exotic physics has been discovered at the LHC so far? We return to this question below, in Sec. III E.

Figure 4 shows the analogous analysis for the $13 \mathrm{TeV}$ data. As compared to the $8 \mathrm{TeV}$ data, the $13 \mathrm{TeV}$ data appear to be less constraining. At first glance, one is tempted to attribute this to low statistics given that the $8 \mathrm{TeV}$ measurement is based on $19.7 \mathrm{fb}^{-1}$ of data while the $13 \mathrm{TeV}$ result is based on $3.2 \mathrm{fb}^{-1}$. However, despite the relatively low statistics at $13 \mathrm{TeV}$, it turns out that the

\footnotetext{
${ }^{4}$ MSTW2008LO PDFs are used only for the purpose of determining the $K$ factor, to be consistent with the calculations for $\sigma_{\mathrm{NNLO}}^{\mathrm{SM}}$ (approx). For all subsequent calculations we use CTEQ6L PDFs.
} 


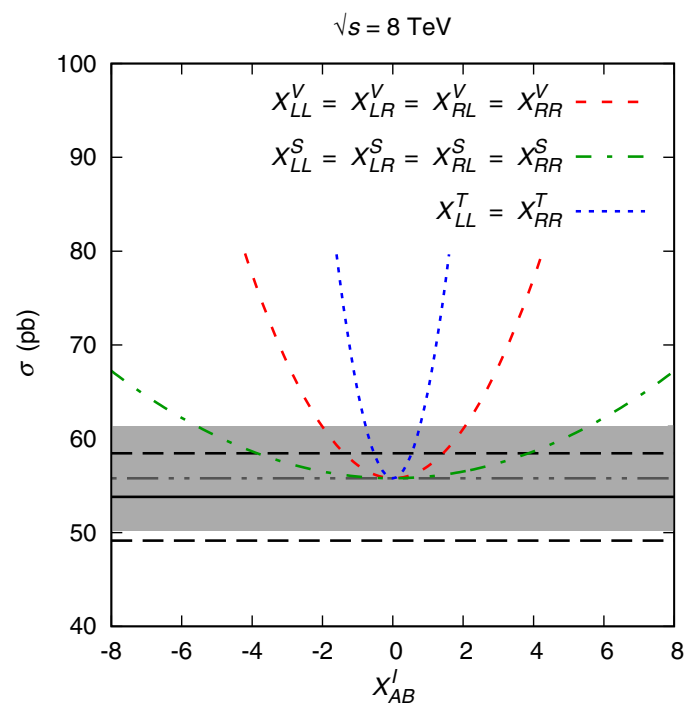

FIG. 3. $t$-channel single-top production cross section at $\sqrt{s}=8 \mathrm{TeV}$. The black solid and dashed horizontal lines depict the CMS measurement [15] along with the total uncertainty. The grey dot-dashed line and the grey shaded region reflect $\sigma_{\mathrm{SM}} \pm 10 \%$, which is calculated as described in the text. The red dashed, green dot-dashed and blue dotted curves give the cross section in the presence of NP vector, scalar and tensor interactions, respectively. As mentioned in the text, only one Lorentz structure is considered at a time.

largest component of the uncertainty is due to systematics. If future analyses can reduce the systematic uncertainty, then tighter constraints can be expected. Presently, for a more effective comparison between the sensitivities to

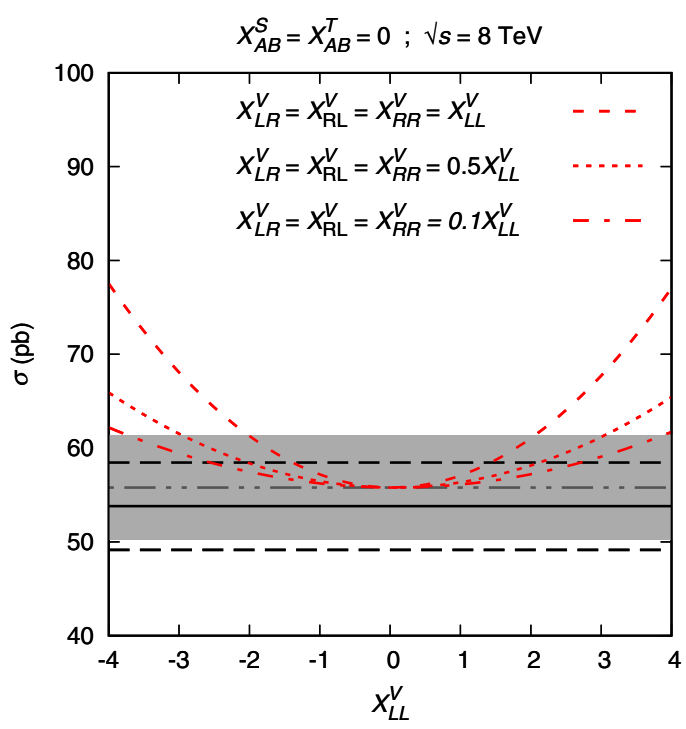

(a)

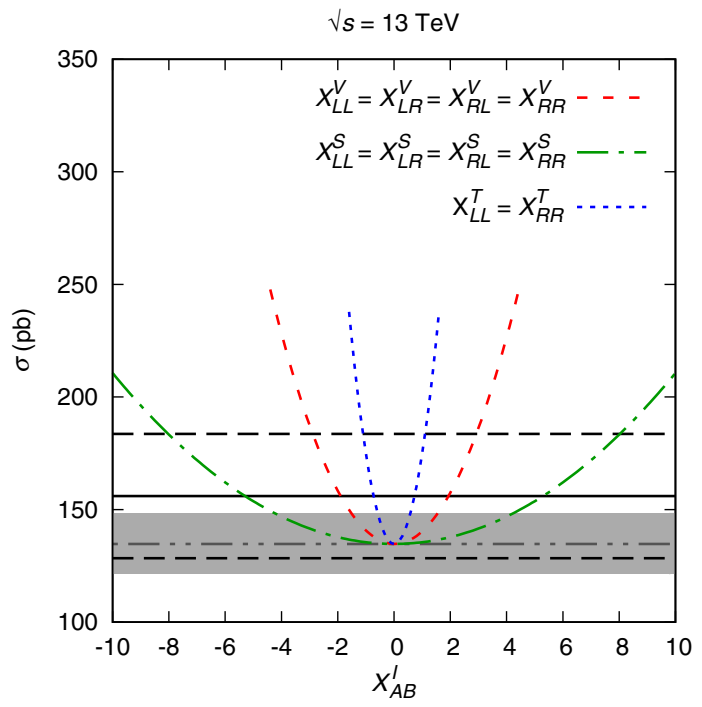

FIG. 4. $t$-channel single-top production cross section at $\sqrt{s}=13 \mathrm{TeV}$. The black solid and dashed horizontal lines depict the ATLAS measurement [17] along with the total uncertainty. The other lines and the shaded region have the same meanings as in Fig. 3.

NP couplings at 8 and $13 \mathrm{TeV}$, we construct a $10 \%$ band around the SM prediction (see Figs. 3 and 4). This gives us an estimate of the improvement in the limits under the assumption that, at both 8 and $13 \mathrm{TeV}$, the central value of the measurement coincides with the SM prediction and has a $10 \%$ uncertainty.

In Figs. 3 and 4, we have allowed all chiral structures associated with a given Lorentz structure to have the

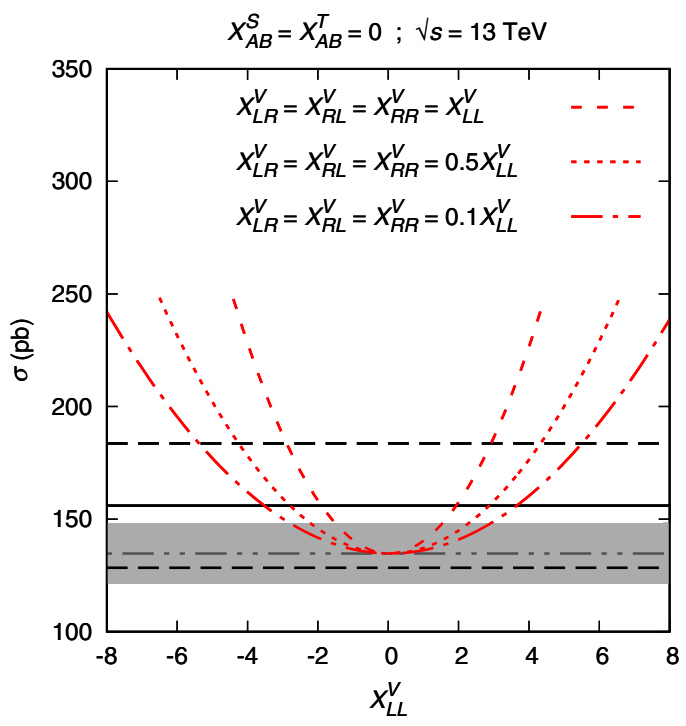

(b)

FIG. 5. $t$-channel single-top production cross section in the presence of various combinations of NP vector contributions at (a) 8 and (b) $13 \mathrm{TeV}$. In the former case, the black solid and dashed horizontal lines depict the CMS measurement [15] along with the total uncertainty; in the latter case, the black solid and dashed horizontal lines depict the ATLAS measurement [17] along with the total uncertainty. In both cases, the grey band depicts $\sigma_{\mathrm{SM}} \pm 10 \%$. 


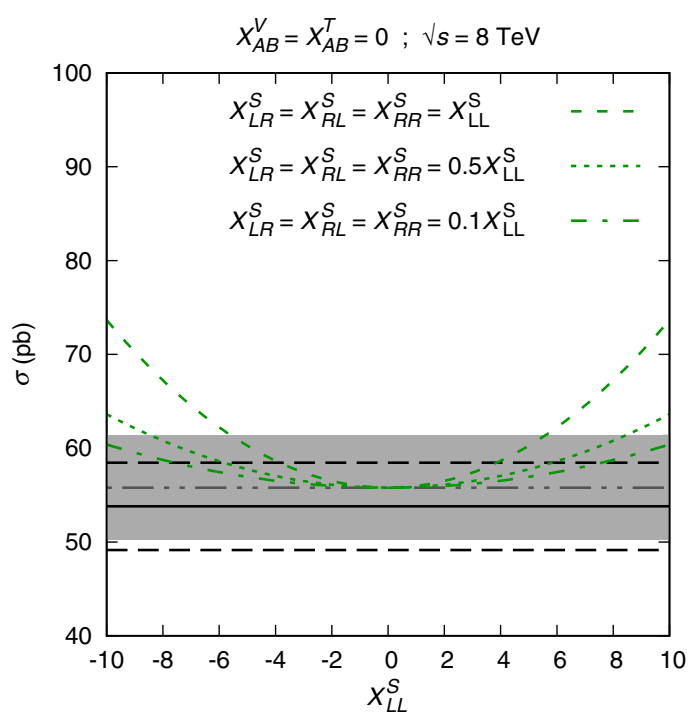

(a)

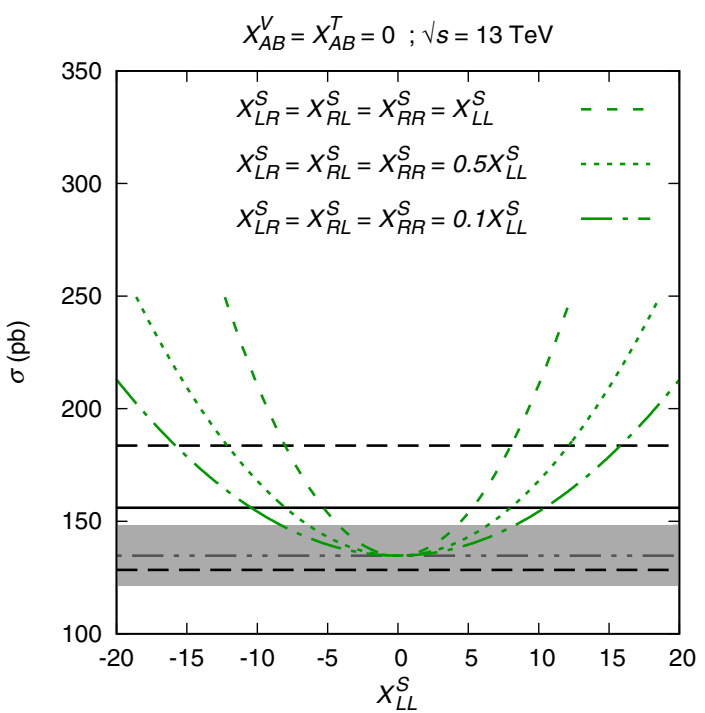

(b)

FIG. 6. $t$-channel single-top production cross section in the presence of NP scalar contributions at (a) 8 and (b) 13 TeV. The horizontal lines and shaded regions have the same meanings as in Fig. 5.

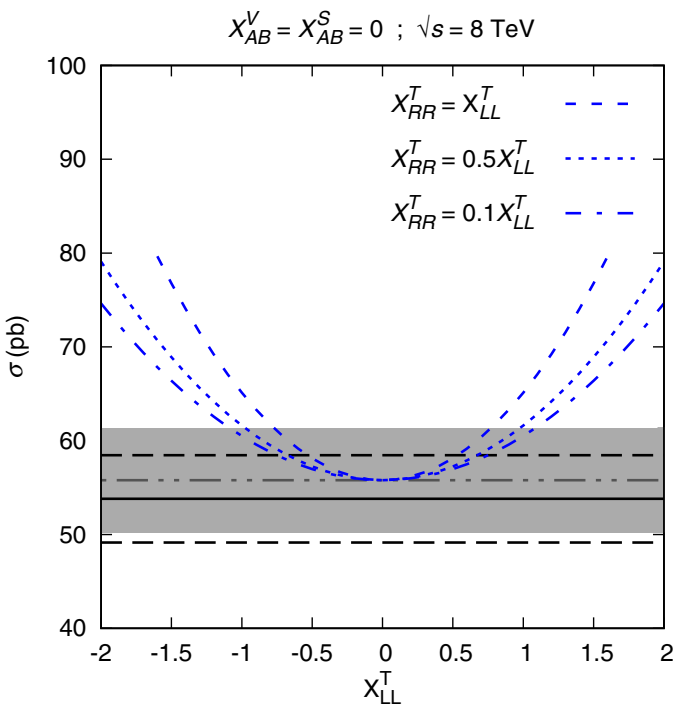

(a)

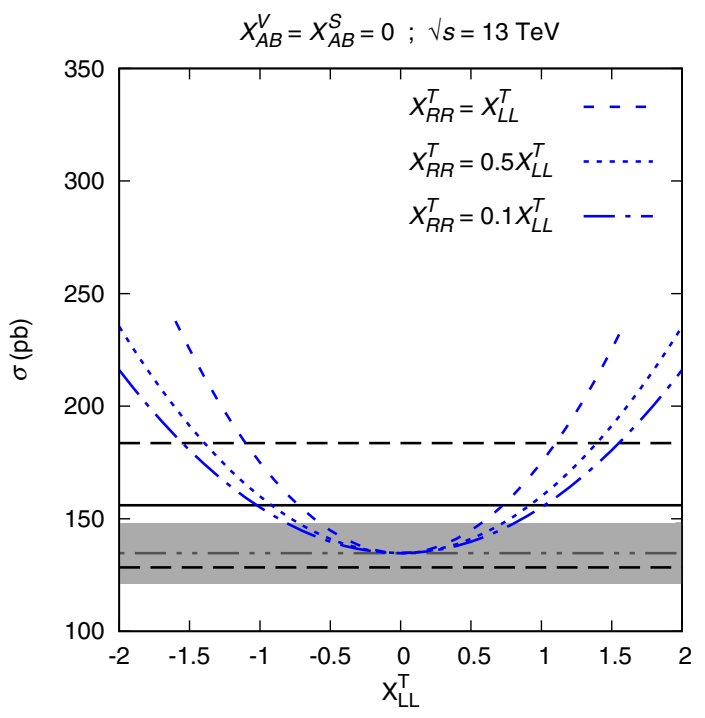

(b)

FIG. 7. $t$-channel single-top production cross section in the presence of NP tensor contributions at (a) 8 and (b) 13 TeV. The horizontal lines and shaded regions have the same meanings as in Fig. 5.

same weight. That is, when vector operators are considered $\left(X_{A B}^{S}=X_{A B}^{T}=0\right)$, we have set $X_{L L}^{V}=X_{L R}^{V}=X_{R L}^{V}=$ $X_{R R}^{V}$, and similarly for scalar and tensor operators. In the following, we relax this condition and consider scenarios where $X_{L R}^{V}, X_{R L}^{V}$ and $X_{R R}^{V}$ are smaller than $X_{L L}^{V}$. As expected, this relaxes the constraint on $X_{L L}^{V}$ (see Fig. 5). In a UV-complete scenario, these operators may not all occur simultaneously and there would exist several possibilities for the relative sizes of the corresponding couplings. We illustrate the effect using one such possibility. The same exercise can be carried out for scalar and tensor operators; the results are depicted in Figs. 6 and 7.

\section{B. $s$-channel single-top production}

Single-top production in the $s$ channel is due to the processes $u_{i} \bar{d}_{j} \rightarrow t \bar{d}_{k}$, with $d_{j, k} \in\{d, s, b\}$ and $u_{i} \in\{u, c\}$. 


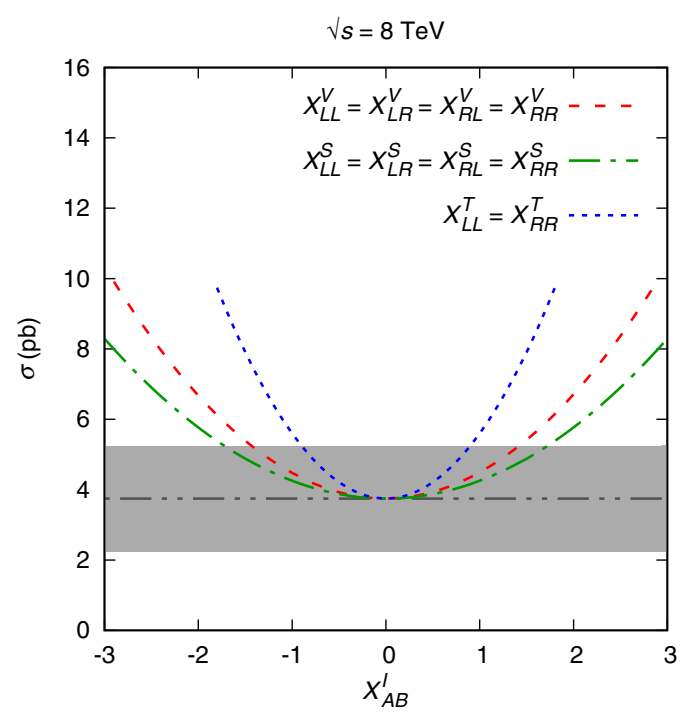

FIG. 8. s-channel single-top production cross section at $\sqrt{s}=8 \mathrm{TeV}$. The grey dot-dashed line and the grey shaded region reflect $\sigma_{\mathrm{SM}} \pm 40 \%$, which is calculated as described in the text. The red dashed, green dot-dashed and blue dotted curves give the cross section in the presence of NP vector, scalar and tensor interactions, respectively. Only one Lorentz structure is considered at a time.

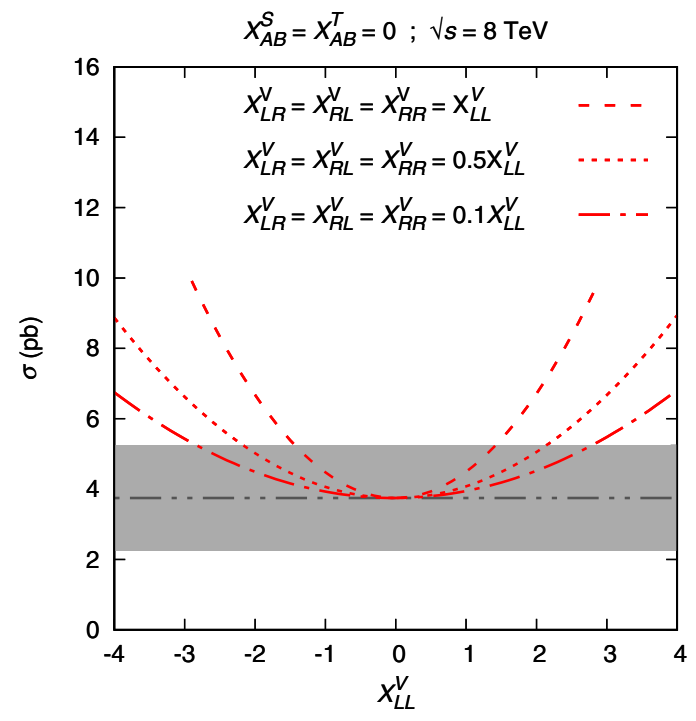

(a)

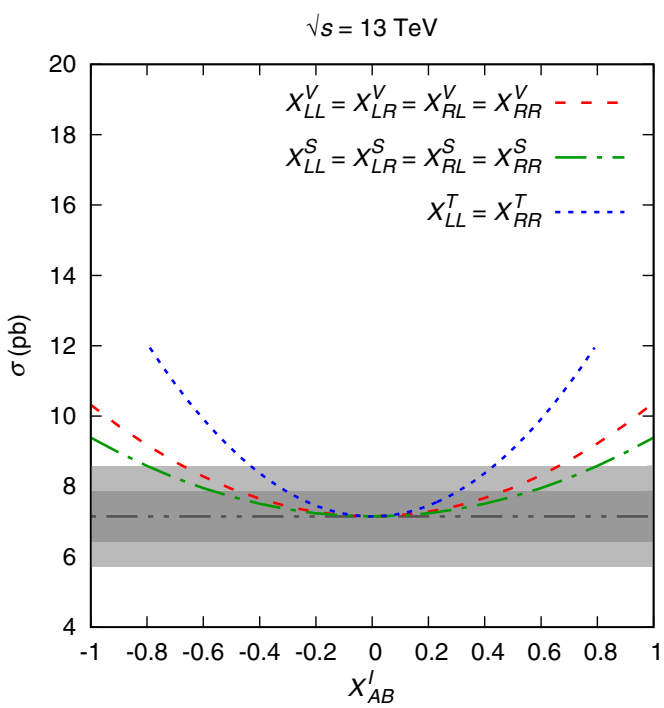

FIG. 9. $s$-channel single-top production cross section at $\sqrt{s}=13 \mathrm{TeV}$. The grey dot-dashed line represents $\sigma_{\mathrm{SM}}$, which is calculated as described in the text. The light-grey and dark-grey shaded regions denote the regions $\sigma_{\mathrm{SM}} \pm 20 \%$ and $\sigma_{\mathrm{SM}} \pm 10 \%$, respectively. The red dashed, green dot-dashed and blue dotted curves give the cross section in the presence of NP vector, scalar and tensor interactions, respectively. As noted above, only one Lorentz structure is considered at a time.

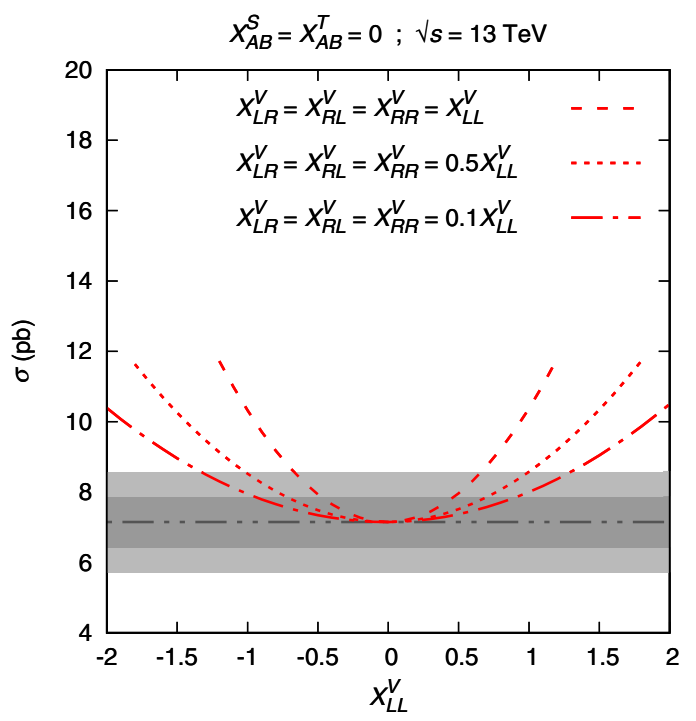

(b)

FIG. 10. $s$-channel single-top production cross section in the presence of NP vector interactions at (a) 8 and (b) 13 TeV. In the former case, the grey band depicts $\sigma_{\mathrm{SM}} \pm 40 \%$; in the latter case, the light-grey band depicts $\sigma_{\mathrm{SM}} \pm 20 \%$ and the dark-grey band depicts $\sigma_{\mathrm{SM}} \pm 10 \%$.

Of these, the largest contribution comes from $u \bar{d} \rightarrow t \bar{b}$, since the rest of the processes are CKM suppressed. However, the introduction of $\mathcal{L}_{\text {eff }}$ enhances the contribution due to $c \bar{b} \rightarrow t \bar{b}$. This is shown in Fig. 8 .
Since the cross section is small $(\sim 3.7 \mathrm{pb}$ in the Standard Model), CMS [16] and ATLAS [13] report the combined cross section due to $p p \rightarrow t X$ and $p p \rightarrow \bar{t} X$. However, in our calculations, we consider 


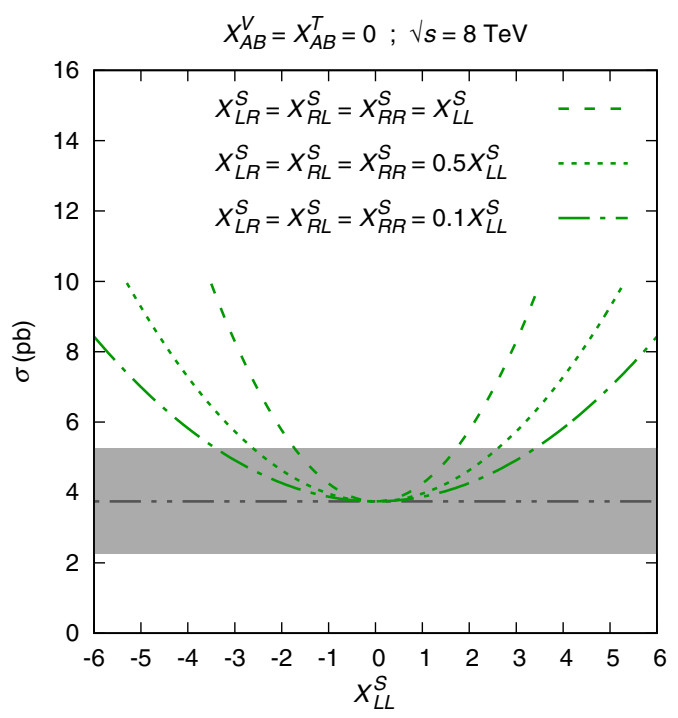

(a)

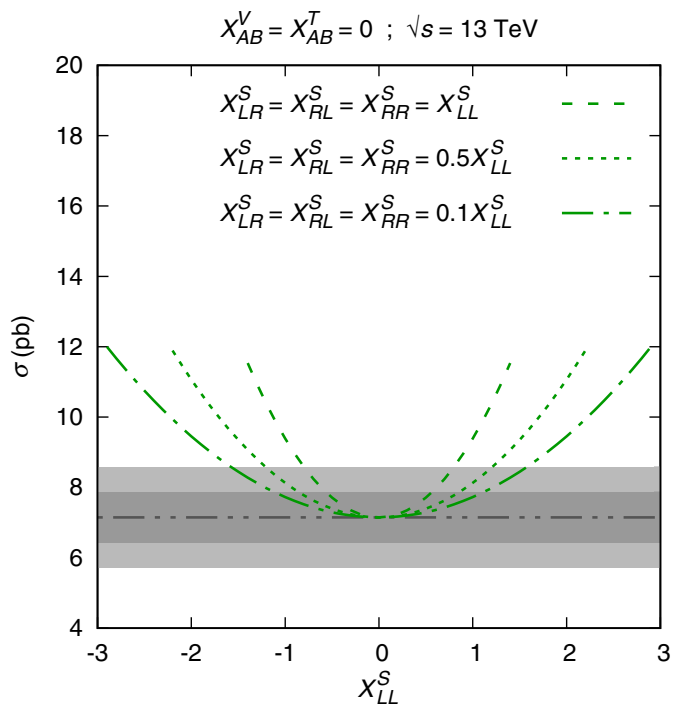

(b)

FIG. 11. $s$-channel single-top production cross section in the presence of NP scalar interactions at (a) 8 and (b) 13 TeV. The grey bands have the same meanings as in Fig. 10.

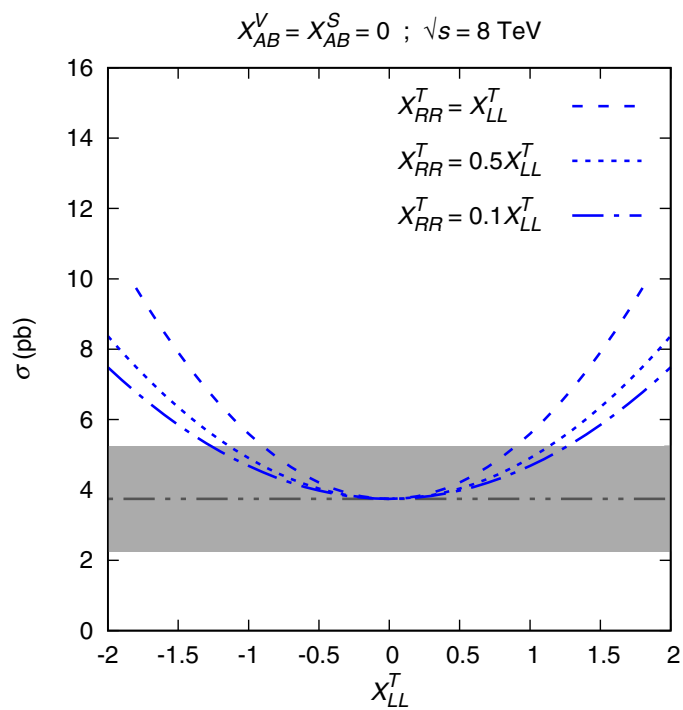

(a)

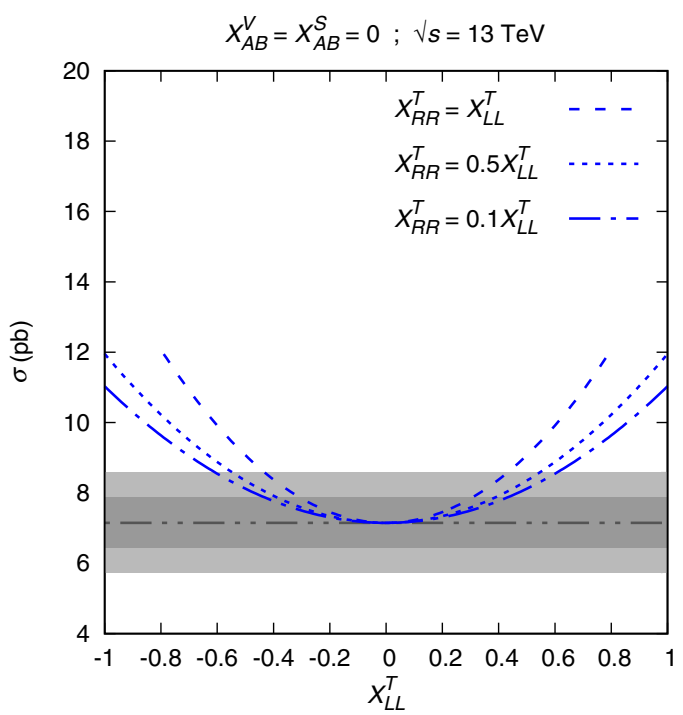

(b)

FIG. 12. $s$-channel single-top production cross section in the presence of NP tensor interactions at (a) 8 and (b) 13 TeV. The grey bands have the same meanings as in Fig. 10.

only $p p \rightarrow t X .^{5}$ Hence, in this section, the experimental measurements are not indicated in the plots. Instead, the grey band denotes a band of uncertainty around the central value of the corresponding SM cross section. The size of the band is chosen so as to be commensurate with the total uncertainty (statistical + systematic) reported in the actual measurement $[13,16]$. In Fig. 8,

\footnotetext{
${ }^{5}$ The NP operators considered in this work do, in fact, contribute to $p p \rightarrow \bar{t} X$; we are, however, restricting our attention to cases in which a single top quark is produced.
}

for example, the size of the band is $\pm 40 \%$. While measurements in the $s$ channel are yet to be reported for $13 \mathrm{TeV}$, this data set is expected to be several times larger than the $8 \mathrm{TeV}$ data set. Hence we use two bands, of sizes $20 \%$ and $10 \%$, to project the limits on $X_{A B}^{I}$ in this case (see Fig. 9).

Comparing Fig. 8 with Fig. 3, it is immediately clear that the $s$-channel process, even when measured with a lower accuracy, yields more stringent bounds than the $t$-channel process. This is easy to understand. $\sigma_{\mathrm{SM}}$ is much smaller for the $s$-channel process than for the $t$-channel 


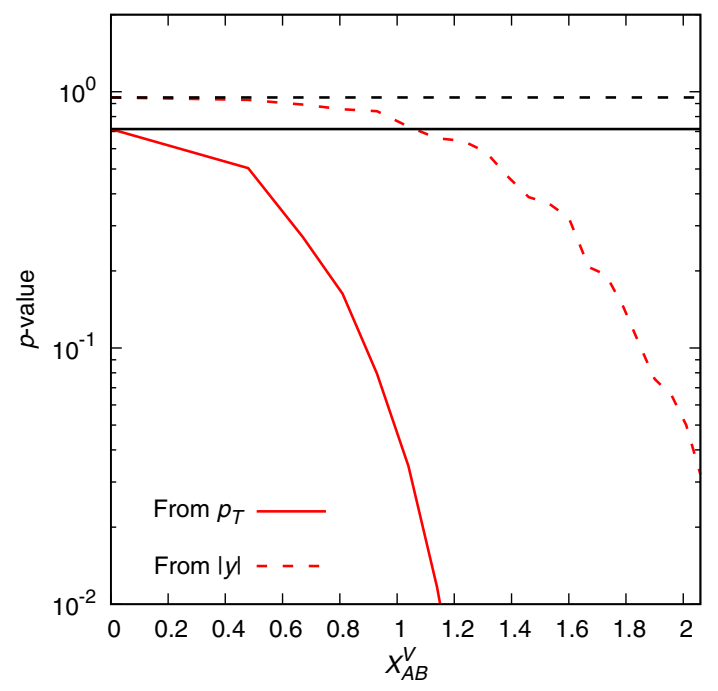

(a)

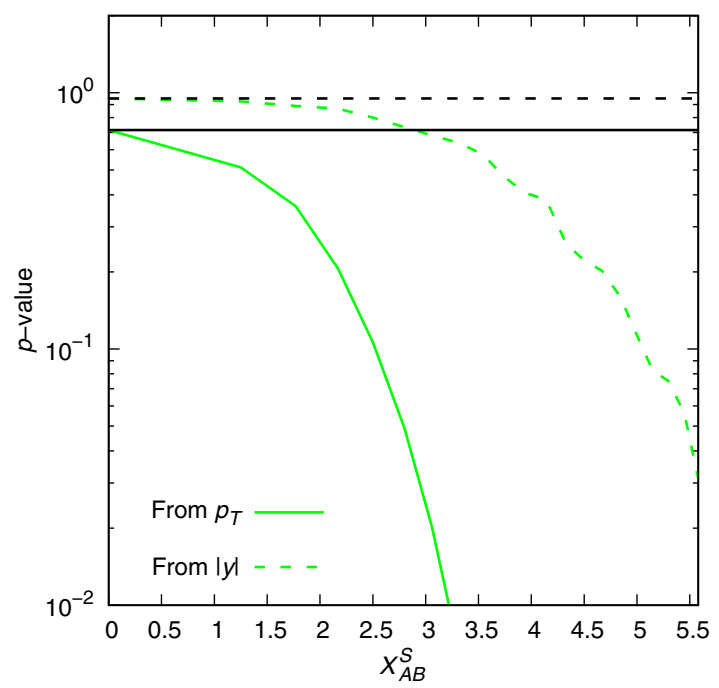

(b)

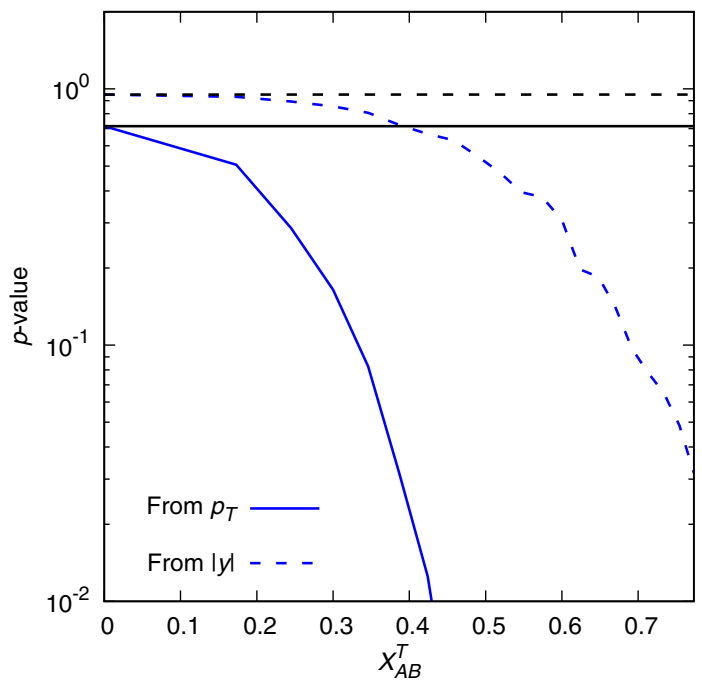

(c)

FIG. 13. $p$-values for various $X_{A B}^{I}$. Once again we assume $X_{L L}^{V}=X_{L R}^{V}=X_{R L}^{V}=X_{R R}^{V}$ in panel (a) and analogously in panels (b) and (c). The solid and dashed lines correspond to the results following from the $p_{T}$ and $|y|$ distributions, respectively. In each case the horizontal line corresponds to the SM result and the other line to the case in which NP is included. See Sec. III C for further details.

process, so even if the relative uncertainty is larger, the absolute deviation allowed is smaller, which leads to tighter constraints on the couplings. A further improvement can be expected to emerge from the $s$-channel measurements at $13 \mathrm{TeV}$ (see Fig. 9). Here again we consider scenarios where one chiral structure is dominant. The impacts on the corresponding limits are shown in Figs. 10-12.

\section{Differential distributions}

The ATLAS and CMS collaborations have measured the differential cross section in terms of the transverse momentum $\left(p_{T}\right)$ and rapidity $(|y|)$ of the top quark for $t$-channel single-top production at $8 \mathrm{TeV}[11,19]$. CMS has also made a similar measurement at $13 \mathrm{TeV}$ [20].
In Sec. III A we identified the ranges of $X_{A B}^{V}, X_{A B}^{S}$ and $X_{A B}^{T}$ that yield a cross section within $10 \%$ of the SM prediction. We now confront the predictions from these NP scenarios ${ }^{6}$ with the ATLAS data [11]. In doing so, we first compute the $\chi^{2}$ by comparing the ATLAS $8 \mathrm{TeV}$ data for the unfolded, parton-level distributions [see Figs. 21(a) and 22(a) and Tables 18 and 20 in Ref. [11]] with the distributions computed for the various NP scenarios. This $\chi^{2}$ is then converted to a $p$-value by the usual formula

\footnotetext{
${ }^{6}$ We now use bin-wise $K$ factors along with our LO computations to estimate the distributions at NLO. The $K$ factors are obtained by comparing the LO predictions for the SM with the PowhEG-BoX+PyThIA6 predictions as given in Ref. [11].
} 


$$
p=\int_{\chi_{\mathrm{obs}}^{2}}^{\infty} f_{\chi^{2}}(x ; n) d x,
$$

where $f_{\chi^{2}}(x ; n)$ is the $\chi^{2}$ probability density function and $n$ is the number of degrees of freedom. The results are plotted in Fig. 13. The $p_{T}$ spectrum proves to have more discriminating power than the $|y|$ spectrum. Using this it is possible to put further constraints on $X_{A B}^{I}$. For example, if we assume that NP scenarios that yield $p<0.05$ are disfavored, then the resulting restriction on $X_{A B}^{V}, X_{A B}^{S}$ and $X_{A B}^{T}$ is shown in Fig. 15 .

\section{Top polarization}

The polarization of the top quark is an important observable at the LHC and a few measurements of it have already been made $[28,29]$. The net polarization is usually defined as

$$
A_{P}=\frac{N_{\uparrow}-N_{\downarrow}}{N_{\uparrow}+N_{\downarrow}}
$$

where $N_{\uparrow}$ and $N_{\downarrow}$, respectively, denote the number of top quarks with spin aligned along or opposite to a chosen direction. The value of $A_{P}$ depends on the choice of the reference direction. The usual choices for this reference direction are the $z$ axis or the momentum of the top itself. In the latter case, $N_{\uparrow}$ and $N_{\downarrow}$ denote the number of top quarks of different helicities. If the top quark is produced in association with another particle (as in the case of single-top production), the momentum of that particle may also be chosen as the reference direction. The utility of $A_{P}$ lies in the fact that it is sensitive to the chiral structure of the coupling at the production vertex. Fortuitously, due to its large mass, the top quark decays before hadronizing. This allows the polarization information (which would otherwise be lost during hadronization) to be gleaned from the angular distribution of the top quark's decay products. Very often, the $e$ or $\mu$ coming from the top decay is used for this purpose.

In the preceding sections, we have identified regions of parameter space that are compatible with various single-top production cross section measurements. We now examine the reach of $A_{P}$ as a means to distinguish between the different types of couplings. In particular, we expect $A_{P}$ to deviate from its Standard Model value when there is an increase in the fraction of $t_{R}$ in the ensemble, that is, when there are contributions from $X_{R L}^{I}$ or $X_{R R}^{I}$. As can be inferred from the expressions in the Appendix, cross section measurements are sensitive to various combinations of sums of $\hat{A}_{i}^{\sigma}$ 's. By way of contrast, the spin-dependent contributions to the amplitude squared are dependent on differences of $\hat{A}_{i}^{\sigma}$ 's, such as $\hat{A}_{\bar{b}}^{+}-\hat{A}_{\bar{b}}^{-}$. For this reason, polarization measurements can yield additional information regarding the types of NP interactions that contribute to the various processes under consideration in this work. In the following
TABLE II. $A_{P}$ for $s$-channel single-top production at $\sqrt{s}=8 \mathrm{TeV}$. The deviation of the cross section from its SM value is of order $20 \%$ in each case.

\begin{tabular}{lcccr}
\hline \hline & $\begin{array}{c}\text { Dominant } \\
\text { Contribution }\end{array}$ & No Contribution & $\sigma(\mathrm{pb})$ & $A_{P}$ \\
\hline SM & $\mathrm{SM}$ & $X_{A B}^{I}$ & 3.7 & -0.68 \\
VP-1 & $X_{L L}^{V}$ & $X_{A B}^{S}, X_{A B}^{T}$ & 4.4 & -0.70 \\
VP-2 & $X_{L R}^{V}$ & $X_{A B}^{S}, X_{A B}^{T}$ & 4.5 & -0.70 \\
VP-3 & $X_{R L}^{V}$ & $X_{A B}^{S}, X_{A B}^{T}$ & 4.5 & -0.43 \\
VP-4 & $X_{R R}^{V}$ & $X_{A B}^{S}, X_{A B}^{T}$ & 4.5 & -0.43 \\
SP-1 & $X_{L L}^{S}$ & $X_{A B}^{V}, X_{A B}^{T}$ & 4.5 & -0.73 \\
SP-2 & $X_{L R}^{S}$ & $X_{A B}^{V}, X_{A B}^{T}$ & 4.5 & -0.73 \\
SP-3 & $X_{R L}^{S}$ & $X_{A B}^{V}, X_{A B}^{T}$ & 4.5 & -0.40 \\
SP-4 & $X_{R R}^{S}$ & $X_{A B}^{V}, X_{A B}^{T}$ & 4.5 & -0.41 \\
TP-1 & $X_{L L}^{T}$ & $X_{A B}^{V}, X_{A B}^{S}$ & 4.5 & -0.65 \\
TP-4 & $X_{R R}^{T}$ & $X_{A B}^{V}, X_{A B}^{S}$ & 4.5 & -0.48 \\
\hline \hline
\end{tabular}

TABLE III. $A_{P}$ for $s$-channel single-top production at $\sqrt{s}=13 \mathrm{TeV}$. The deviation of the cross section from its SM value is of order $10 \%$ in each case.

\begin{tabular}{lcccr}
\hline \hline & $\begin{array}{c}\text { Dominant } \\
\text { Contribution }\end{array}$ & No Contribution & $\sigma(\mathrm{pb})$ & $A_{P}$ \\
\hline SM & $\mathrm{SM}$ & $X_{A B}^{I}$ & 7.1 & -0.67 \\
VP-1 & $X_{L L}^{V}$ & $X_{A B}^{S}, X_{A B}^{T}$ & 7.8 & -0.69 \\
VP-2 & $X_{L R}^{V}$ & $X_{A B}^{S}, X_{A B}^{T}$ & 7.8 & -0.69 \\
VP-3 & $X_{R L}^{V}$ & $X_{A B}^{S}, X_{A B}^{T}$ & 7.8 & -0.55 \\
VP-4 & $X_{R R}^{V}$ & $X_{A B}^{S}, X_{A B}^{T}$ & 7.8 & -0.53 \\
SP-1 & $X_{L L}^{S}$ & $X_{A B}^{V}, X_{A B}^{T}$ & 7.8 & -0.71 \\
SP-2 & $X_{L R}^{S}$ & $X_{A B}^{V}, X_{A B}^{T}$ & 7.8 & -0.71 \\
SP-3 & $X_{R L}^{S}$ & $X_{A B}^{V}, X_{A B}^{T}$ & 7.8 & -0.54 \\
SP-4 & $X_{R R}^{S}$ & $X_{A B}^{V}, X_{A B}^{T}$ & 7.8 & -0.52 \\
TP-1 & $X_{L L}^{T}$ & $X_{A B}^{V}, X_{A B}^{S}$ & 7.9 & -0.65 \\
TP-4 & $X_{R R}^{T}$ & $X_{A B}^{V}, X_{A B}^{S}$ & 7.9 & -0.56 \\
\hline \hline
\end{tabular}

analysis, we choose certain values of $X_{A B}^{I}$ that yield a cross section within a certain "allowed" range and then compute $A_{P}$ according to Eq. (6) above, with $N_{\uparrow}$ and $N_{\downarrow}$ denoting the number of top quarks of either helicity. ${ }^{7}$ Note that we include NP effects in both the numerator and the denominator when calculating $A_{P}$.

Table II lists $s$-channel single-top polarization asymmetries for various combinations of NP contributions at $\sqrt{s}=8 \mathrm{TeV}$. In choosing parameters, we have allowed for an enhancement of $20 \%$ in the cross section over the SM prediction, noting that the experimental uncertainty is close to $40 \%$. We consider scenarios for which the

\footnotetext{
${ }^{7}$ That is, the reference axis is given by the top quark's momentum.
} 


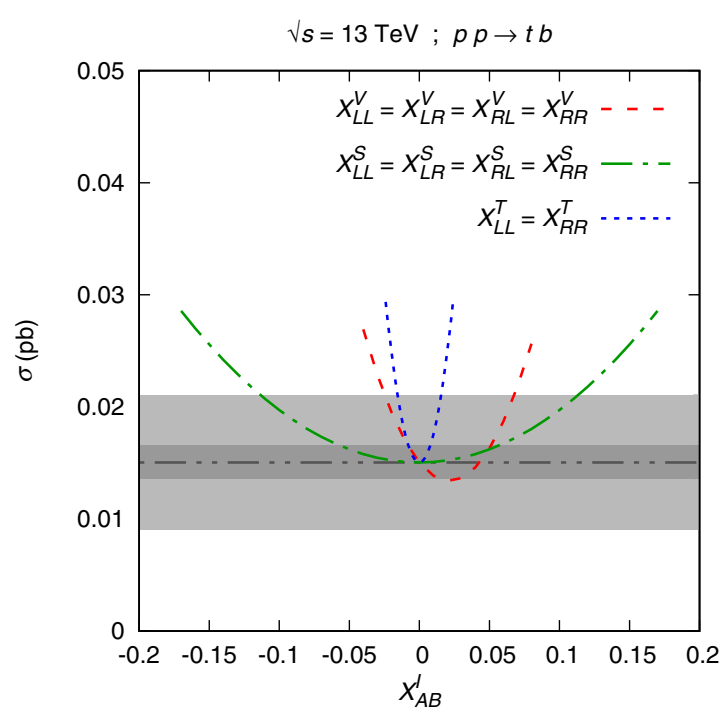

(a)

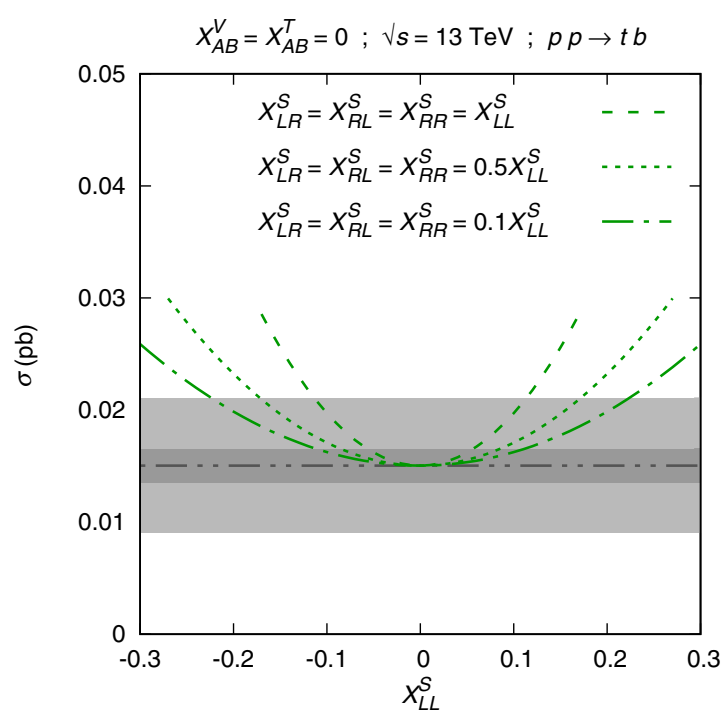

(c)

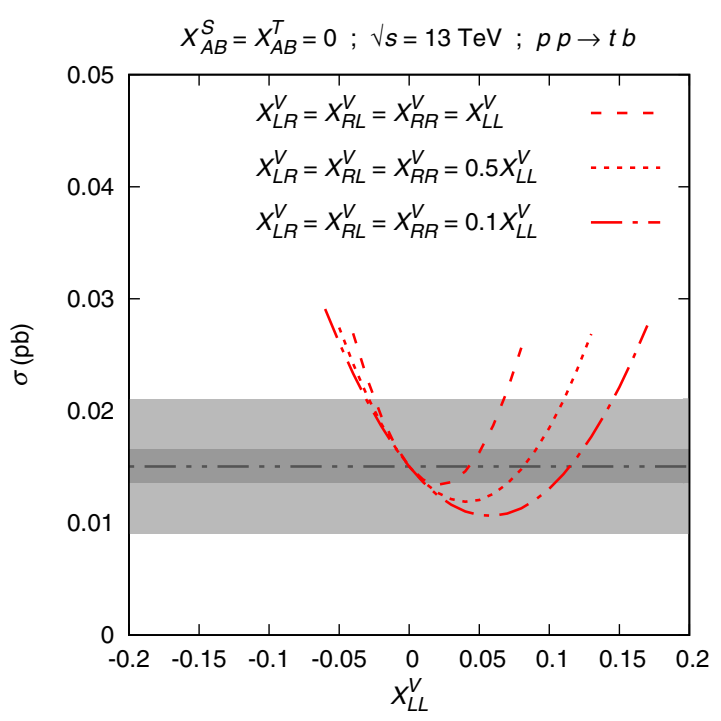

(b)

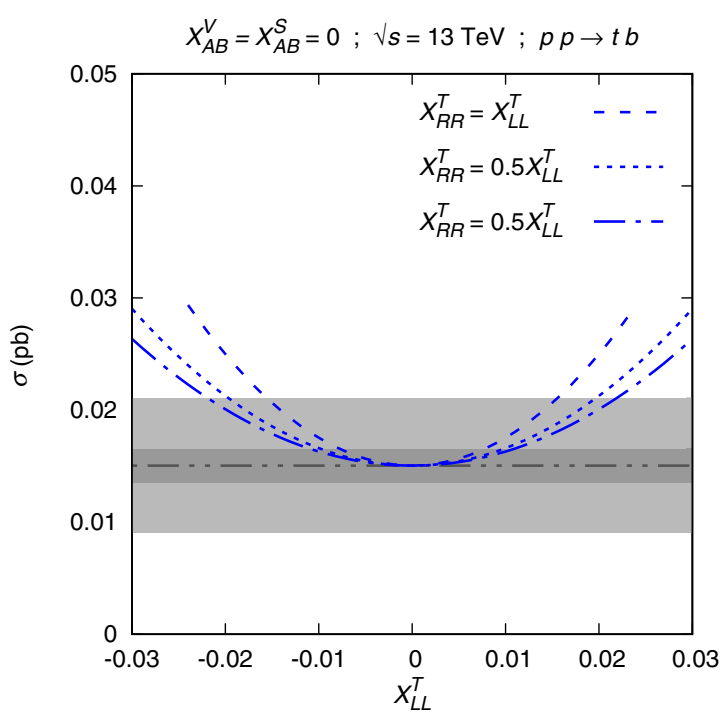

(d)

FIG. 14. Single-top production cross section in the channel $p p \rightarrow t b$ in the presence of $X_{A B}^{I}$ at 13 TeV. The light-grey band depicts $\sigma_{\mathrm{SM}} \pm 40 \%$, while the dark-grey band depicts $\sigma_{\mathrm{SM}} \pm 10 \%$.

dominant contribution comes from one operator, ${ }^{8}$ keeping the subdominant couplings at approximately one-tenth of the dominant one. We find that the deviation in $A_{P}$ can be considerable. Note that the values of $A_{P}$ listed in Table II are based on the calculation of tree-level cross sections, as described at the beginning of Sec. III. $K$ factors cancel out in the calculation of $A_{P}$. The estimation of $A_{P}$ based on higher-order calculations is beyond the scope of this work. However, the dominant higher-order corrections would arise from QCD effects and, as such, they are not expected to alter $A_{P}$ significantly.

\footnotetext{
${ }^{8}$ This is also more likely from the point of view of a UV-complete model.
}

We have not attempted to estimate the accuracy with which the $A_{P}$ can be measured at the LHC. ${ }^{9}$ We note, however, that a recent analysis from CMS [29] was able to make a measurement with approximately $38 \%$ uncertainty. We also point out that the results from Ref. [29] cannot be used to compare directly with our results because their choice of reference axis is different from ours. Nonetheless, it is clear from Table II that accuracies $\sim 10 \%$ or better would be needed in order for $A_{P}$ to be a useful discriminator.

\footnotetext{
${ }^{9}$ We refrain from making such an attempt, since background rejection depends heavily on the specific algorithm used, which, in turn, often involves boosted decision trees [29] and other sophisticated analysis tools developed and trained by experimentalists to get the best from their respective detectors.
} 


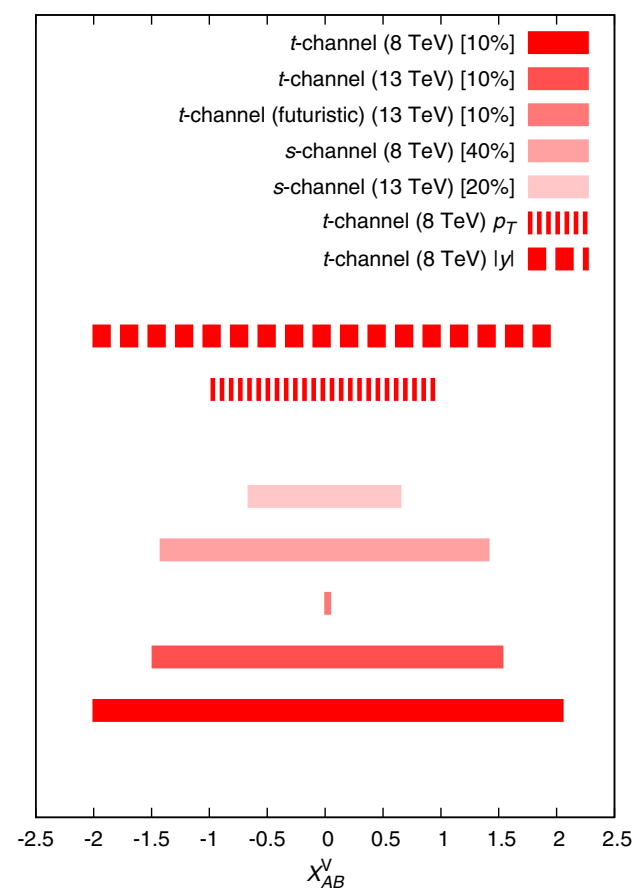

(a)

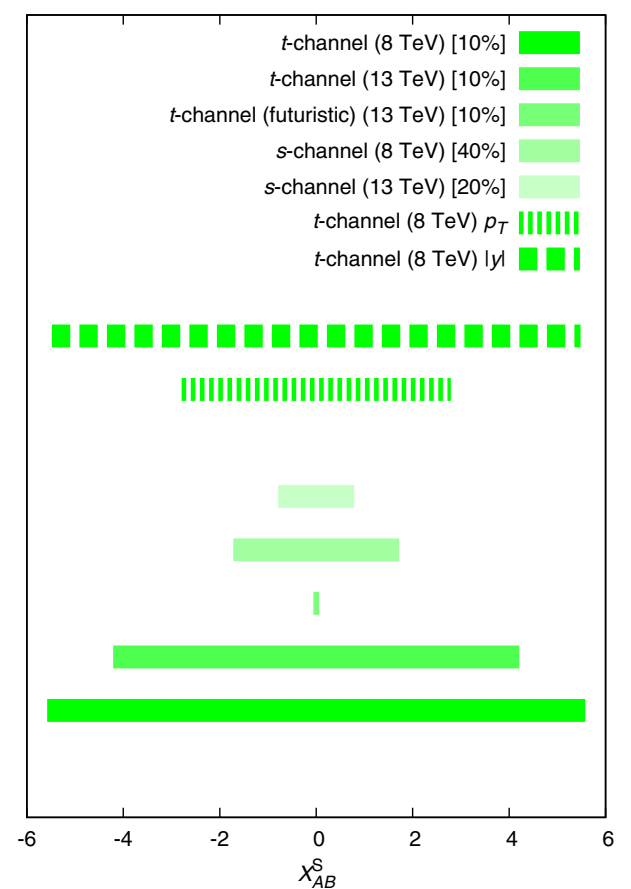

(b)

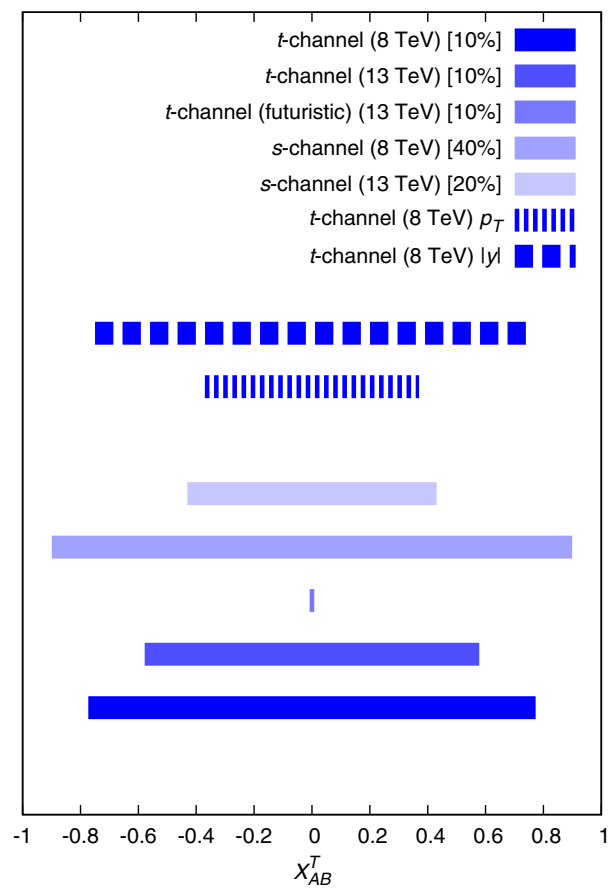

(c)

FIG. 15. Comparison between the limits obtained on (a) $X_{A B}^{V}$, (b) $X_{A B}^{S}$ and (c) $X_{A B}^{T}$ from the different channels. The numbers in square brackets indicate the allowed deviation from the SM cross section, e.g., $10 \%$ indicates $\Delta \sigma=0.1 \sigma_{\mathrm{SM}}$. For panel (a) we set $X_{L L}^{V}=X_{L R}^{V}=X_{R L}^{V}=X_{R R}^{V}$, and similarly for panels (b) and (c). Also shown are the limits obtained by imposing the condition $p>0.05$ on the $p_{T}$ and $|y|$ distributions. For details, see Secs. III A, III B, III C and III E.

Finally, in Table III, we list the polarization asymmetry for $s$-channel single-top production at $\sqrt{s}=13 \mathrm{TeV}$. This time we allow for $\sim 10 \%$ enhancement in the cross section over the SM prediction. Once again the largest deviations correspond to contributions from $X_{R L}^{I}$ and $X_{R R}^{I}$, although the net size of the deviation is smaller. This is largely an 
artifact of the restriction placed on the overall increase in cross section; allowing for a larger variation in the cross section would, in general, allow for greater deviation in $A_{P}$. It would not, however, guarantee a greater deviation in $A_{P}$, since the size of $A_{P}$ depends on the chiral structure of the dominant operator.

One can, similarly, obtain the polarization asymmetry for $t$-channel single-top production. If we follow our earlier strategy of choosing benchmark points from well within the allowed band, we would be considering scenarios where $\Delta \sigma \sim 0.05 \sigma_{\mathrm{SM}}$. In that case, the deviation in $A_{P}$ is $\lesssim 10 \%$ and may not be experimentally discernible. However, as one allows for larger $\Delta \sigma$, deviations in $A_{P}$ also begin to increase. Tables similar to Tables II and III for the $t$ channel are not presented here for the sake of brevity.

\section{E. $t$-channel single-top production: A futuristic analysis}

We now return to the futuristic analysis that we briefly alluded to in the Introduction. As discussed in Sec. III A, $t$-channel single-top production gets contributions from all processes of the type $d_{i} u_{j} \rightarrow t d_{k}$ and $d_{i} \bar{d}_{j} \rightarrow t \bar{u}_{k}$ where $u_{j, k} \in\{u, c\}$ and $d_{i, j, k} \in\{d, s, b\}$. The new physics operators, however, only affect the subprocesses $b c \rightarrow t b$ and $b \bar{b} \rightarrow t \bar{c}$. If one of these subprocesses could be isolated and studied separately, then it would be possible to obtain far more stringent constraints on the new physics parameters. The reason for the enormous increase in sensitivity is obvious: in the earlier cases, the NP contribution arising from just two subprocesses had to compete with the SM background arising from a multitude of subprocesses. In this case, it would be competing with background arising from just one subprocess. This also resolves the conundrum that we encountered in Sec. III A: if $X_{A B}^{I}$ of $\mathcal{O}(1)$ (or, equivalently $\Lambda_{\text {new }} \lesssim 1 \mathrm{TeV}$ ) are not ruled out, why has new physics not been discovered at the LHC? It is because the sensitivity of the LHC in this context is limited by the fact that one or two NP amplitudes have to compete against a large number of SM amplitudes.

From the point of view of isolating $t$-channel subprocesses, $b c \rightarrow t b$ appears at first glance to be more promising than $b \bar{b} \rightarrow t \bar{c}$, since $b c \rightarrow t b$ events could be isolated from other $t$-channel events by the application of an additional $b$ tag. In reality, the situation is somewhat complicated.

In order to identify $s$-channel single-top production and distinguish it from $t$-channel single-top production, experiments already use an additional $b$ tag. The idea here is that if a top quark is produced in an $s$-channel process, then about $99 \%$ of the time, it is accompanied by a bottom quark owing to the strength of $V_{t b}$. In contrast, a $t b$ final state is rare in the $t$-channel process. ${ }^{10}$ Since $b$ tags do not distinguish between

\footnotetext{
${ }^{10} \mathrm{~A} t d$ final state is approximately $10^{4}$ times more likely to occur than a $t b$ final state in $t$-channel single-top production.
}

$b$ and $\bar{b}$, both $t b$ and $t \bar{b}$ final states are identified as coming from the $s$-channel process. The contamination in $s$-channel measurements arising out of such misidentifications is not significant, since the cross section for the ( $t$-channel) $t b$ final state is orders of magnitude smaller than the dominant $s$-channel contribution.

However, if it were possible to distinguish between $b$ and $\bar{b}$ quarks, then one would be able to isolate the process $b c \rightarrow t b$. To estimate the expected improvement in the limits, one only needs to consider the size of the SM background, which, at $13 \mathrm{TeV}$, is approximately $135 \mathrm{pb}$ for the usual $t$-channel production and about $0.014 \mathrm{pb}$ when $b c \rightarrow t b$ is isolated. The actual limits are depicted in Fig. 14, which can be compared with Fig. 4 and with the $13 \mathrm{TeV}$ plots in Figs. 5-7. $\sigma_{\mathrm{SM}} \pm 10 \%$ is depicted as a grey band in each of these cases. In Figs. 14(a) and 14(b), the effect of the interference between the SM and the NP $X_{L L}^{V}$ term is discernible, unlike in the corresponding plots in Sec. III A.

While isolating $b c \rightarrow t b$ seems to be an exciting possibility, techniques for distinguishing between $b$-flavored quarks and antiquarks in the final state are still at a nascent stage, although some developments in this direction have been reported [30]. If such techniques can be improved upon sufficiently so as to become reliable even when the statistics are relatively low, then the $p p \rightarrow t b$ channel will become the primary channel of interest.

\section{SUMMARY AND CONCLUSIONS}

Top quark decays are most sensitive to new physics effects at the energy scale of a few hundred GeV. The same effects, if they arise from a higher energy scale, would be more effectively probed in single-top production. In this work, we have focused on NP effects arising from anomalous couplings between the top, bottom and charm quarks. Since we only considered the top's interactions with heavy quarks, it might at first appear that progress would be thwarted by the low densities of heavy quarks inside the proton. Nevertheless, our detailed study shows that it would be possible to place meaningful constraints on the new physics parameters.

We have considered $t$-channel and $s$-channel single-top production cross sections to obtain constraints on contact interactions involving $t, b$ and $c$ quarks. Of these two channels, the stronger constraints arise from the $s$ channel. This is due to the fact that the Standard Model background cross section is smaller for the $s$ channel. Within a given channel ( $t$ or $s$ ), the limits are most stringent for tensor operators, followed by vector and scalar operators, respectively. This is essentially due to the additional numerical factors that appear from the Dirac traces for each of these operators.

For $t$-channel single-top production, data is also available for the $p_{T}$ and $|y|$ differential cross sections. We have examined the compatibility of these measurements with 
different NP scenarios and found that the $p_{T}$ distribution in particular can be useful in further constraining the NP parameter space.

Apart from the total and differential cross sections, we have also considered the relative contributions to the cross section from top quarks of different helicities. The polarization asymmetry $A_{P}$, which compares the helicity states of the top quark, can be particularly useful in establishing the presence of operators involving $t_{R}$, especially since the corresponding Standard Model charged current coupling involves only $t_{L}$.

Finally, we have considered a futuristic analysis. This analysis rests on one crucial assumption, namely that $b$ quarks in the final state can be distinguished from $\bar{b}$ antiquarks on an event-by-event basis. Adopting this assumption, we have obtained limits on the NP four-quark operators that are far more stringent than those obtained from the regular $t$-channel and $s$-channel analyses. The comparison can be seen in Fig. 15. The improvement is indeed startling and perhaps adequate motivation for the pursuit of experimental techniques that will make it possible.

\section{ACKNOWLEDGMENTS}

The authors wish to thank David Cusick and Kristian Stephens for collaboration at early stages of this work, and David London and Bhubanjyoti Bhattacharya for helpful discussions. We are most grateful to the ATLAS Collaboration for acceding to our request for additional information. We would also like to thank the referee for pointing out additional experimental results relevant to this analysis. This work has been partially supported by Consejo Nacional de Investigaciones Científicas y Técnicas
(CONICET) and Agencia Nacional de Promoción Científica y Tecnológica (ANPCyT) projects No. PICT2013-2266 and No. PICT-2016-0164 (A. S.). K. K. was supported by the United States National Science Foundation under Grant No. PHY-1215785. The work of P. S. was supported by the Department of Science and Technology, India under the INSPIRE Faculty Scheme Grant No. IFA14-PH-105.

\section{APPENDIX: PARTON-LEVEL MATRIX ELEMENTS SQUARED}

In this appendix we write down parton-level expressions for the matrix element squared for the $s$ - and $t$-channel processes considered in this paper. Throughout, we use the notation $Q^{2}=\left(p_{1}+p_{2}\right)^{2}$ and $q^{2}=\left(p_{1}-p_{3}\right)^{2}$. Also, the spin four-vector for the top quark may be written as [31]

$$
s_{t}^{\mu}=\left(\frac{\vec{p}_{t} \cdot \hat{s}_{t}}{m_{t}}, \hat{s}_{t}+\frac{\left(\vec{p}_{t} \cdot \hat{s}_{t}\right) \vec{p}_{t}}{m_{t}\left(E_{t}+m_{t}\right)}\right),
$$

where $\vec{p}_{t}$ and $E_{t}$ represent the top quark's three-momentum and energy (in a given reference frame), and where $\hat{s}_{t}$ is a unit vector defined in the rest frame of the top quark. Note that $p_{t} \cdot s_{t}=0$, as expected. The symbol $\bar{\Sigma}$ denotes that spin and color are averaged for the initial state and summed for the final state, except for the spin of the final-state top quark. In each case,

$$
\bar{\Sigma}|\mathcal{M}|^{2}=\bar{\Sigma}\left|\mathcal{M}_{\mathrm{SM}}\right|^{2}+\bar{\Sigma}\left|\mathcal{M}_{\mathrm{NP}}\right|^{2}+2 \operatorname{Re}\left(\bar{\Sigma} \mathcal{M}_{\mathrm{NP}}^{\dagger} \mathcal{M}_{\mathrm{SM}}\right) .
$$

Finally, we adopt the shorthand notation $\epsilon\left(p_{1}, p_{2}, p_{4}, s_{t}\right) \equiv$ $\epsilon^{\alpha \beta \lambda \eta} p_{1 \alpha} p_{2 \beta} p_{4 \lambda} s_{t \eta}$, taking $\epsilon^{0123}=+1$.

\section{1. s-channel: $\bar{b}\left(p_{1}\right) c\left(p_{2}\right) \rightarrow t\left(p_{3}, s_{t}\right) \bar{b}\left(p_{4}\right)$}

$$
\begin{aligned}
\bar{\Sigma}\left|\mathcal{M}_{\mathrm{SM}}\right|^{2}= & 16 G_{F}^{2} M_{W}^{4} \frac{\left|V_{t b}\right|^{2}\left|V_{c b}\right|^{2}}{\left(Q^{2}-M_{W}^{2}\right)^{2}+\Gamma_{W}^{2} M_{W}^{2}}\left[\left(p_{2} \cdot p_{4}\right) p_{1} \cdot\left(p_{3}-m_{t} s_{t}\right)\right], \\
\bar{\Sigma}\left|\mathcal{M}_{\mathrm{NP}}\right|^{2}= & 16 G_{F}^{2}\left|V_{c b}\right|^{2}\left|V_{t b}\right|^{2} \\
& \times\left[\left(p_{1} \cdot p_{3}\right)\left(p_{2} \cdot p_{4}\right)\left(\hat{A}_{\bar{b}}^{+}+\hat{A}_{\bar{b}}^{-}\right)+\left(p_{1} \cdot p_{4}\right)\left(p_{2} \cdot p_{3}\right)\left(\hat{A}_{c}^{+}+\hat{A}_{c}^{-}\right)\right. \\
& +\left(p_{1} \cdot p_{2}\right)\left(p_{3} \cdot p_{4}\right)\left(\hat{A}_{b}^{+}+\hat{A}_{b}^{-}\right)-m_{t}\left(p_{2} \cdot p_{4}\right)\left(p_{1} \cdot s_{t}\right)\left(\hat{A}_{\bar{b}}^{+}-\hat{A}_{\bar{b}}^{-}\right) \\
& -m_{t}\left(p_{1} \cdot p_{4}\right)\left(p_{2} \cdot s_{t}\right)\left(\hat{A}_{c}^{+}-\hat{A}_{c}^{-}\right)-m_{t}\left(p_{1} \cdot p_{2}\right)\left(p_{4} \cdot s_{t}\right)\left(\hat{A}_{b}^{+}-\hat{A}_{b}^{-}\right) \\
& \left.-8 m_{t} \operatorname{Im}\left(X_{L L}^{T} X_{L L}^{S *}+X_{R R}^{T} X_{R R}^{S *}\right) \epsilon\left(p_{1}, p_{2}, p_{4}, s_{t}\right)\right], \\
2 \operatorname{Re}\left(\bar{\Sigma} \mathcal{M}_{\mathrm{NP}}^{\dagger} \mathcal{M}_{\mathrm{SM}}\right)= & 64 G_{F}^{2} M_{W}^{2}\left|V_{t b}\right|^{2}\left|V_{c b}\right|^{2} \frac{\left[\operatorname{Re}\left(X_{L L}^{V}\right)\left(Q^{2}-M_{W}^{2}\right)-\operatorname{Im}\left(X_{L L}^{V}\right) \Gamma_{W} M_{W}\right]}{\left(Q^{2}-M_{W}^{2}\right)^{2}+\Gamma_{W}^{2} M_{W}^{2}} \\
& \times\left[\left(p_{2} \cdot p_{4}\right) p_{1} \cdot\left(p_{3}-m_{t} s_{t}\right)\right] .
\end{aligned}
$$




$$
\begin{aligned}
& \text { 2. } \boldsymbol{t} \text {-channel: } \boldsymbol{b}\left(\boldsymbol{p}_{\mathbf{1}}\right) \boldsymbol{c}\left(\boldsymbol{p}_{\mathbf{2}}\right) \rightarrow \boldsymbol{t}\left(\boldsymbol{p}_{\mathbf{3}}, \boldsymbol{s}_{\boldsymbol{t}}\right) \boldsymbol{b}\left(\boldsymbol{p}_{\mathbf{4}}\right) \text { [32] } \\
\bar{\Sigma}\left|\mathcal{M}_{\mathrm{SM}}\right|^{2}= & 16 G_{F}^{2} M_{W}^{4} \frac{\left|V_{t b}\right|^{2}\left|V_{c b}\right|^{2}}{\left(q^{2}-M_{W}^{2}\right)^{2}}\left[\left(p_{1} \cdot p_{2}\right) p_{4} \cdot\left(p_{3}-m_{t} s_{t}\right)\right], \\
\bar{\Sigma}\left|\mathcal{M}_{\mathrm{NP}}\right|^{2}= & 16 G_{F}^{2}\left|V_{c b}\right|^{2}\left|V_{t b}\right|^{2} \\
& \times\left[\left(p_{1} \cdot p_{2}\right)\left(p_{3} \cdot p_{4}\right)\left(\hat{A}_{\bar{b}}^{+}+\hat{A}_{\bar{b}}^{-}\right)+\left(p_{1} \cdot p_{4}\right)\left(p_{2} \cdot p_{3}\right)\left(\hat{A}_{c}^{+}+\hat{A}_{c}^{-}\right)\right. \\
& +\left(p_{1} \cdot p_{3}\right)\left(p_{2} \cdot p_{4}\right)\left(\hat{A}_{b}^{+}+\hat{A}_{b}^{-}\right)-m_{t}\left(p_{1} \cdot p_{2}\right)\left(p_{4} \cdot s_{t}\right)\left(\hat{A}_{\bar{b}}^{+}-\hat{A}_{\bar{b}}^{-}\right) \\
& -m_{t}\left(p_{1} \cdot p_{4}\right)\left(p_{2} \cdot s_{t}\right)\left(\hat{A}_{c}^{+}-\hat{A}_{c}^{-}\right)-m_{t}\left(p_{2} \cdot p_{4}\right)\left(p_{1} \cdot s_{t}\right)\left(\hat{A}_{b}^{+}-\hat{A}_{b}^{-}\right) \\
& \left.+8 m_{t} \operatorname{Im}\left(X_{L L}^{T} X_{L L}^{S *}+X_{R R}^{T} X_{R R}^{S *}\right) \epsilon\left(p_{1}, p_{2}, p_{4}, s_{t}\right)\right], \\
2 \operatorname{Re}\left(\bar{\Sigma} \mathcal{M}_{\mathrm{NP}}^{\dagger} \mathcal{M}_{\mathrm{SM}}\right)= & 64 G_{F}^{2} M_{W}^{2}\left(\frac{\left|V_{t b}\right|^{2}\left|V_{c b}\right|^{2} \operatorname{Re}\left(X_{L L}^{V}\right)}{q^{2}-M_{W}^{2}}\right)\left[\left(p_{1} \cdot p_{2}\right) p_{4} \cdot\left(p_{3}-m_{t} s_{t}\right)\right] .
\end{aligned}
$$

\section{3. $t$-channel: $b\left(p_{1}\right) \bar{b}\left(p_{2}\right) \rightarrow t\left(p_{3}, s_{t}\right) \bar{c}\left(p_{4}\right)$}

$$
\begin{aligned}
\bar{\Sigma}\left|\mathcal{M}_{\mathrm{SM}}\right|^{2}= & 16 G_{F}^{2} M_{W}^{4} \frac{\left|V_{t b}\right|^{2}\left|V_{c b}\right|^{2}}{\left(q^{2}-M_{W}^{2}\right)^{2}}\left[\left(p_{1} \cdot p_{4}\right) p_{2} \cdot\left(p_{3}-m_{t} s_{t}\right)\right], \\
\bar{\Sigma}\left|\mathcal{M}_{\mathrm{NP}}\right|^{2}= & 16 G_{F}^{2}\left|V_{c b}\right|^{2}\left|V_{t b}\right|^{2} \\
& \times\left[\left(p_{1} \cdot p_{4}\right)\left(p_{2} \cdot p_{3}\right)\left(\hat{A}_{\bar{b}}^{+}+\hat{A}_{\bar{b}}^{-}\right)+\left(p_{1} \cdot p_{2}\right)\left(p_{3} \cdot p_{4}\right)\left(\hat{A}_{c}^{+}+\hat{A}_{c}^{-}\right)\right. \\
& +\left(p_{1} \cdot p_{3}\right)\left(p_{2} \cdot p_{4}\right)\left(\hat{A}_{b}^{+}+\hat{A}_{b}^{-}\right)-m_{t}\left(p_{1} \cdot p_{4}\right)\left(p_{2} \cdot s_{t}\right)\left(\hat{A}_{\bar{b}}^{+}-\hat{A}_{\bar{b}}\right) \\
& -m_{t}\left(p_{1} \cdot p_{2}\right)\left(p_{4} \cdot s_{t}\right)\left(\hat{A}_{c}^{+}-\hat{A}_{c}^{-}\right)-m_{t}\left(p_{2} \cdot p_{4}\right)\left(p_{1} \cdot s_{t}\right)\left(\hat{A}_{b}^{+}-\hat{A}_{b}^{-}\right) \\
& \left.-8 m_{t} \operatorname{Im}\left(X_{L L}^{T} X_{L L}^{S *}+X_{R R}^{T} X_{R R}^{S *}\right) \epsilon\left(p_{1}, p_{2}, p_{4}, s_{t}\right)\right], \\
2 \operatorname{Re}\left(\bar{\Sigma} \mathcal{M}_{\mathrm{NP}}^{\dagger} \mathcal{M}_{\mathrm{SM}}\right)= & 64 G_{F}^{2} M_{W}^{2}\left(\frac{\left|V_{t b}\right|^{2}\left|V_{c b}\right|^{2} \operatorname{Re}\left(X_{L L}^{V}\right)}{q^{2}-M_{W}^{2}}\right)\left[\left(p_{1} \cdot p_{4}\right) p_{2} \cdot\left(p_{3}-m_{t} s_{t}\right)\right] .
\end{aligned}
$$

[1] T. A. Aaltonen et al. (CDF and D0 Collaborations), Phys. Rev. Lett. 120, 042001 (2018).

[2] R. Aaij et al. (LHCb Collaboration), Phys. Rev. Lett. 113, 151601 (2014).

[3] R. Aaij et al. (LHCb Collaboration), J. High Energy Phys. 02 (2016) 104; A. Abdesselam et al. (Belle Collaboration), arXiv:1604.04042.

[4] R. Aaij et al. (LHCb Collaboration), J. High Energy Phys. 09 (2015) 179.

[5] J. P. Lees et al. (BABAR Collaboration), Phys. Rev. D 88, 072012 (2013); R. Aaij et al. (LHCb Collaboration), Phys. Rev. Lett. 115, 111803 (2015); 115, 159901(E) (2015).

[6] K. Kiers, T. Knighton, D. London, M. Russell, A. Szynkman, and K. Webster, Phys. Rev. D 84, 074018 (2011); K. Kiers, P. Saha, A. Szynkman, D. London, S. Judge, and J. Melendez, Phys. Rev. D 90, 094015 (2014); P. Saha, K. Kiers, D. London, and A. Szynkman, Phys. Rev. D 90, 094016 (2014); P. Saha, K. Kiers, B. Bhattacharya, D. London, A. Szynkman, and J. Melendez, Phys. Rev. D 93, 054044 (2016).
[7] T. Han, M. Hosch, K. Whisnant, B. L. Young, and X. Zhang, Phys. Rev. D 58, 073008 (1998); T. M. P. Tait and C.-P. Yuan, Phys. Rev. D 63, 014018 (2000); J. A. Aguilar-Saavedra, Nucl. Phys. B804, 160 (2008).

[8] R. Goldouzian and B. Clerbaux, arXiv:1612.01795; D. Pinna, A. Zucchetta, M. R. Buckley, and F. Canelli, Phys. Rev. D 96, 035031 (2017); X. Y. Jiang, J. Z. Han, G. Yang, and C.X. Yu, Commun. Theor. Phys. 67, 415 (2017); I. Ahmed, arXiv:1711.08348; P. Pani and G. Polesello, Phys. Dark Universe 21, 8 (2018).

[9] P. M. Ferreira, O. Oliveira, and R. Santos, Phys. Rev. D 73, 034011 (2006); P. M. Ferreira and R. Santos, Phys. Rev. D 73, 054025 (2006); 74, 014006 (2006); R. A. Coimbra, P. M. Ferreira, R. B. Guedes, O. Oliveira, A. Onofre, R. Santos, and M. Won, Phys. Rev. D 79, 014006 (2009); J. A. Aguilar-Saavedra, Nucl. Phys. B843, 638 (2011); B851, 443(E) (2011); G. Durieux, F. Maltoni, and C. Zhang, Phys. Rev. D 91, 074017 (2015); J. A. AguilarSaavedra, C. Degrande, and S. Khatibi, Phys. Lett. B 769, 498 (2017). 
[10] G. Aad et al. (ATLAS Collaboration), Phys. Rev. D 90, 112006 (2014).

[11] M. Aaboud et al. (ATLAS Collaboration), Eur. Phys. J. C 77, 531 (2017).

[12] ATLAS Collaboration, Report No. ATLAS-CONF-2011-118.

[13] G. Aad et al. (ATLAS Collaboration), Phys. Lett. B 756, 228 (2016); 740, 118 (2015).

[14] S. Chatrchyan et al. (CMS Collaboration), J. High Energy Phys. 12 (2012) 035.

[15] V. Khachatryan et al. (CMS Collaboration), J. High Energy Phys. 06 (2014) 090.

[16] V. Khachatryan et al. (CMS Collaboration), J. High Energy Phys. 09 (2016) 027.

[17] M. Aaboud et al. (ATLAS Collaboration), J. High Energy Phys. 04 (2017) 086.

[18] A. M. Sirunyan et al. (CMS Collaboration), Phys. Lett. B 772, 752 (2017).

[19] CMS Collaboration, Report No. CMS-PAS-TOP-14-004.

[20] CMS Collaboration, Report No. CMS-PAS-TOP-16-004.

[21] LHC Top Working Group, https://twiki.cern.ch/twiki/bin/ view/LHCPhysics/LHCTopWGSummaryPlots.

[22] J. Alwall, M. Herquet, F. Maltoni, O. Mattelaer, and T. Stelzer, J. High Energy Phys. 06 (2011) 128.
[23] A. Alloul, N. D. Christensen, C. Degrande, C. Duhr, and B. Fuks, Comput. Phys. Commun. 185, 2250 (2014).

[24] J. Pumplin, D. R. Stump, J. Huston, H. L. Lai, P. M. Nadolsky, and W. K. Tung, J. High Energy Phys. 07 (2002) 012.

[25] A. D. Martin, W. J. Stirling, R. S. Thorne, and G. Watt, Eur. Phys. J. C 63, 189 (2009).

[26] N. Kidonakis, Phys. Rev. D 81, 054028 (2010).

[27] N. Kidonakis, arXiv:1205.3453; arXiv:1311.0283.

[28] S. Chatrchyan et al. (CMS Collaboration), Phys. Rev. Lett. 112, 182001 (2014); V. Khachatryan et al. (CMS Collaboration), Phys. Rev. D 93, 052007 (2016); G. Aad et al. (ATLAS Collaboration), Phys. Rev. Lett. 111, 232002 (2013).

[29] V. Khachatryan et al. (CMS Collaboration), J. High Energy Phys. 04 (2016) 073.

[30] V. M. Abazov et al. (D0 Collaboration), Phys. Rev. Lett. 98, 041801 (2007); G. Aad et al. (ATLAS Collaboration), J. High Energy Phys. 11 (2013) 031.

[31] C. Itzykson and J. B. Zuber, Quantum Field Theory (McGraw-Hill, New York, 1980); K. Kiers, A. Szynkman, and D. London, Phys. Rev. D 74, 035004 (2006).

[32] D. Cusick and K. Stephens (private communication). 